\title{
Weak Markovian Bisimilarity: Abstracting from Prioritized/Weighted Internal Immediate Actions
}

\author{
Marco Bernardo and Alessandro Aldini \\ Università di Urbino "Carlo Bo" - Italy
}

\begin{abstract}
Markovian process calculi constitute a useful framework for reasoning about the functional and performance aspects of concurrent systems. This is achieved by means of behavioral equivalences that take into account both the action names and their exponentially distributed durations. A notable extension to the expressiveness of Markovian process calculi derives from the adoption of GSPNlike immediate actions, i.e. actions with a zero duration and equipped with a priority level and a weight. Since internal immediate actions are unobservable both from the functional viewpoint and from the performance viewpoint, in this paper we tackle the problem of defining a weak variant of Markovian bisimilarity that abstracts from such actions. We show that the proposed equivalence is a congruence and admits a sound and complete axiomatization for the class of well-prioritized process terms.
\end{abstract}

\section{Introduction}

Markovian process calculi have been developed as performance-oriented extensions of traditional calculi (see, e.g., Refs. 4,6,7). In essence, they introduce exponentially distributed delays governed by the race policy, can be equipped with behavioral equivalences that take into account such stochastic delays besides the action names, and provide continuous-time Markov chain (CTMC) models for performance evaluation purposes.

When using pure Markovian process calculi to model concurrent systems in practice, the availability of only exponentially distributed delays may be too restrictive. Although proper combinations of exponential distributions (called phase-type distributions) can approximate most of the general distributions arbitrarily closely, some useful distributions are left out, specially the one representing a zero duration. The capability of expressing zero durations would also constitute a good performance abstraction mechanism, similar to the internal action name $\tau$ on the functional side. 
In the modeling process it often happens to deal with choices among logical events (like the reception of a message vs. its loss) with which no timing can reasonably be associated, or to encounter activities that are several orders of magnitude faster than the activities that are important for the evaluation of certain performance measures. In all of these cases, using a zero duration would be an adequate solution from the modeling standpoint, but this is not permitted by pure Markovian process calculi.

In order to manage zero durations, it is necessary to extend the syntax of Markovian process calculi and, consequently, their underlying CTMC models. In the literature, this has been accomplished in three different ways.

The first one has been to separate action names from time using two distinct prefix operators - one for action names and one for exponentially distributed delays - and to consider the execution of an action name to take zero time. ${ }^{6}$ This is the result of the combination of nondeterministic process calculi and CTMCs, which thus yields CTMC models extended with nondeterministic branchings.

The second way has been to introduce a probabilistic choice operator, by means of which it is possible to preselect a certain group of exponentially timed actions that are enabled. ${ }^{10}$ As a consequence, this technique yields CTMC models extended with probabilistic branchings. Precisely, the proposed model requires a strict alternation between probabilistic branchings and Markovian branchings, with any probabilistic transition ending in a state having - besides possible exponentially timed transitions - a single probabilistic transition labeled with 1 that goes back to the state itself.

The third way has been to admit immediate actions as first-class citizens in the syntax, ${ }^{3}$ in the same way as immediate transitions are first-class citizens within generalized stochastic Petri net (GSPN) models. ${ }^{1}$ Besides a name and a zero duration, each immediate action has associated with it a priority and a weight, which are used to choose among several immediate actions that are simultaneously enabled. Therefore, this technique yields CTMC models extended with prioritized/probabilistic branchings, which freely alternate with Markovian branchings.

The third technique can thus be viewed as a complementary variant of the first one in which nondeterminism is ruled out by augmenting each action name with a priority and a weight. It can also be regarded as an extension of the second one in which the choice operator is made both prioritized and probabilistic and the alternation constraint is relaxed.

Zero durations are negligible from the performance viewpoint in the same way as internal actions are negligible from the functional viewpoint. 
As a consequence, in each of the three extended Markovian frameworks mentioned above, any entity that is internal and takes zero time is absolutely unobservable. This should be taken into account when developing behavioral equivalences in those frameworks. Actually, this has already been done both in Ref. 6 and in Ref. 10, where two weak variants of Markovian bisimilarity ${ }^{7}$ have been defined.

The problem that we address here is the development of a weak variant of Markovian bisimilarity for a Markovian process calculus - like EMPA ${ }_{\mathrm{gr}}{ }^{4}$ - extended with immediate actions à la GSPN. The technical difficulty lies in the fact that the objective of abstracting from internal immediate actions is complicated by the need of retaining information about the priorities and the weights of such actions. Moreover, we shall see that it is necessary to distinguish among observable, initially unobservable and fully unobservable process terms, and that congruence and axiomatization can be achieved only for a class of process terms that we shall call well prioritized.

Besides being of theoretical interest, this extension of Markovian bisimilarity would be useful also from a practical viewpoint, as it would increase the number of system models that can be compared/manipulated. As an example, consider a queueing system ${ }^{8}$ with two classes of customers. Such customers arrive at the service center according to a Poisson process, then compete for the resources on the basis of their priorities (assume that customers of the second class take precedence over those of the first class). The service center is composed of two buffers - one for each class - and a number of servers that provide a service to the customers of both classes at a certain rate. The activities that are important from the performance viewpoint are the arrivals and the services, while the delivery of a customer from the buffer reserved for its class to one of the servers has a negligible duration. Therefore the delivery activities are the ones that could be abstracted away if viewed as being internal to the service center, so that in the end the queueing system could be viewed as being equivalent to a suitable birth-death process. The point is that the delivery activities necessarily carry over priority information, which cannot be handled with the weak variants of Markovian bisimilarity defined in Refs. 6 and 10.

This paper is organized as follows. In Sect. 2 we revisit EMPAgr. In Sect. 3 we define a weak variant of Markovian bisimilarity over EMPAgr and we show that it is a congruence and admits a sound and complete axiomatization for the class of well-prioritized process terms. In Sect. 4 we provide some concluding remarks. The proofs of all results can be found at http://www.sti.uniurb.it/bernardo/. 


\section{Extended Markovian Process Algebra}

In this section we revisit $\mathrm{EMPA}_{\mathrm{gr}},{ }^{4}$ a Markovian process algebra extended with prioritized/weighted immediate actions à la $\mathrm{GSPN}^{1}$ in which interprocess communication is based on a multiway generative-reactive ${ }^{5}$ synchronization mechanism.

In $\mathrm{EMPA}_{\text {gr }}$ every action is durational, hence it is represented as a pair $<a, \tilde{\lambda}>$, where $a$ is the action name and $\tilde{\lambda}$ is the action rate. There are three kinds of actions: exponentially timed, immediate, and passive.

Exponentially timed actions are of the form $\left\langle a, \lambda>\right.$ with $\lambda \in \mathbb{R}_{>0}$. The duration of each such action is exponentially distributed with parameter equal to the action rate (hence its average duration is the inverse of its rate). Whenever several exponentially timed actions are enabled, the race policy is adopted, hence the fastest action is the one that is executed. As a consequence, the execution probability of an exponentially timed action is proportional to its rate.

Immediate actions are of the form $\left\langle a, \infty_{l, w}\right\rangle$, where $l \in \mathbf{N}_{>0}$ is the priority level and $w \in \mathbb{R}_{>0}$ is the weight. Each immediate action has duration zero and takes precedence over exponentially timed actions (which are assumed to have priority level 0 ). Whenever several immediate actions are enabled, the generative preselection policy is adopted, i.e. each action is given an execution probability proportional to its weight provided that it has the highest priority level within the considered action set.

Passive actions are of the form $\left\langle a, *_{w}^{l}\right\rangle$, where $l \in \mathrm{N}$ is the priority constraint and $w \in \mathbb{R}_{>0}$ is the weight. Each passive action with priority constraint $l$ can synchronize only with another passive action having the same name and the same priority constraint, or with a non-passive action having the same name and priority level $l$. Whenever several passive actions are enabled, the reactive preselection policy is adopted, i.e. within every set of enabled passive actions with the same name and the same priority constraint each action is given an execution probability proportional to its weight. As a consequence, the choice between two passive actions with different names or different priority constraints is nondeterministic.

While in Ref. 4 each passive action had a reactive priority level associated with it, here passive actions are assigned priority constraints. These come into play in the synchronization of a passive action with a non-passive one by forcing the priority level of the latter to be equal to the priority constraint of the former. Priority constraints will be useful to achieve congruence for our weak Markovian bisimilarity, as they allow the interplay of the priorities of actions of different components to be kept under control. 
Definition 2.1. Let $A c t=$ Name $\times$ Rate be a set of actions, with Name being a set of action names containing a distinguished symbol $\tau$ for the invisible action and Rate $=\mathbb{R}_{>0} \cup\left\{\infty_{l, w} \mid l \in \mathbf{N}_{>0} \wedge w \in \mathbf{R}_{>0}\right\} \cup\left\{*_{w}^{l} \mid\right.$ $\left.l \in \mathbf{N} \wedge w \in \mathbb{R}_{>0}\right\}$ being a set of action rates (ranged over by $\tilde{\lambda}$ ). Let Relab $=\left\{\varphi:\right.$ Name $\longrightarrow$ Name $\left.\mid \varphi^{-1}(\tau)=\{\tau\}\right\}$ be a set of action name relabeling functions that preserve visibility. The set of the process terms of $\mathrm{EMPA}_{\mathrm{gr}}$ is generated by the following syntax:

$$
P::=\underline{0}|<a, \tilde{\lambda}>. P| P+P|P / L| P[\varphi]\left|P \|_{S} P\right| A
$$

where $L, S \subseteq$ Name $-\{\tau\}$ and $A$ is a process constant defined through the (possibly recursive) equation $A \triangleq P$.

The semantics for the set $\mathcal{P}$ of the closed and guarded process terms

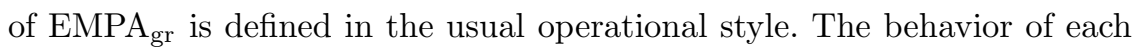
term $P$ is given by a multitransition system $\llbracket P \rrbracket$, whose states correspond to process terms and whose transitions - each of which has a multiplicity equal to the number of proofs of its derivation - are labeled with actions.

The null term $\underline{0}$ cannot execute any action, hence the corresponding labeled multitransition system is just a state with no transitions.

The action prefix term $<a, \tilde{\lambda}>. P$ can execute an action with name $a$ and rate $\tilde{\lambda}$ and then behaves as $P$ :

$$
<a, \lambda>. P \stackrel{a, \lambda}{\longrightarrow} P \quad<a, \infty_{l, w}>. P \stackrel{a, \infty_{l, w}}{\longrightarrow} P \quad<a, *_{w}^{l}>. P \stackrel{a, *_{w}^{l}}{\longrightarrow} P
$$

The alternative composition $P_{1}+P_{2}$ behaves as either $P_{1}$ or $P_{2}$ depending on whether $P_{1}$ or $P_{2}$ executes an action first:

$$
\frac{P_{1} \stackrel{a, \tilde{\lambda}}{\longrightarrow} P^{\prime}}{P_{1}+P_{2} \stackrel{a, \tilde{\lambda}}{\longrightarrow} P^{\prime}} \quad \frac{P_{2} \stackrel{a, \tilde{\lambda}}{\longrightarrow} P^{\prime}}{P_{1}+P_{2} \stackrel{a, \tilde{\lambda}}{\longrightarrow} P^{\prime}}
$$

The hiding term $P / L$ behaves as $P$ with the difference that the name of every action executed by $P$ that belongs to $L$ is turned into $\tau$ :

$$
\frac{P \stackrel{a, \tilde{\lambda}}{\longrightarrow} P^{\prime} \quad a \in L}{P / L \stackrel{\tau, \tilde{\lambda}}{\longrightarrow} P^{\prime} / L} \quad \frac{P \stackrel{a, \tilde{\lambda}}{\longrightarrow} P^{\prime} \quad a \notin L}{P / L \stackrel{a, \tilde{\lambda}}{\longrightarrow} P^{\prime} / L}
$$

The relabeling term $P[\varphi]$ behaves as $P$ with the difference that the name $a$ of every action executed by $P$ is turned into $\varphi(a)$ :

$$
\frac{P \stackrel{a, \tilde{\lambda}}{\longrightarrow} P^{\prime}}{P[\varphi] \stackrel{\varphi(a), \tilde{\lambda}}{\longrightarrow} P^{\prime}[\varphi]}
$$


The parallel composition $P_{1} \|_{S} P_{2}$ behaves as $P_{1}$ in parallel with $P_{2}$ as long as actions are executed whose name does not belong to $S$ :

$$
\frac{P_{1} \stackrel{a, \tilde{\lambda}}{\longrightarrow} P_{1}^{\prime} \quad a \notin S}{P_{1}\left\|_{S} P_{2} \stackrel{a, \tilde{\lambda}}{\longrightarrow} P_{1}^{\prime}\right\|_{S} P_{2}} \quad \frac{P_{2} \stackrel{a, \tilde{\lambda}}{\longrightarrow} P_{2}^{\prime} \quad a \notin S}{P_{1}\left\|_{S} P_{2} \stackrel{a, \tilde{\lambda}}{\longrightarrow} P_{1}\right\|_{S} P_{2}^{\prime}}
$$

Synchronizations are forced between any non-passive action executed by one term and any passive action executed by the other term that have the same name belonging to $S$ and the same priority level/constraint:

$$
\begin{aligned}
& \stackrel{P_{1} \stackrel{a, \lambda}{\longrightarrow} P_{1}^{\prime} \quad P_{2} \stackrel{a, *_{w}^{0}}{\longrightarrow} P_{2}^{\prime} \quad a \in S}{P_{1}\left\|_{S} P_{2} \stackrel{a, \lambda \cdot \frac{w}{\text { weight }^{w}\left(P_{2}, a, 0\right)}}{\longrightarrow} P_{1}^{\prime}\right\|_{S} P_{2}^{\prime}} \quad \frac{P_{1} \stackrel{a, *_{w}^{0}}{\longrightarrow} P_{1}^{\prime} \quad P_{2} \stackrel{a, \lambda}{\longrightarrow} P_{2}^{\prime} \quad a \in S}{P_{1}\left\|_{S} P_{2} \stackrel{a, \lambda \cdot \frac{w}{\text { weight }\left(P_{1}, a, 0\right)}}{\longrightarrow} P_{1}^{\prime}\right\|_{S} P_{2}^{\prime}}
\end{aligned}
$$

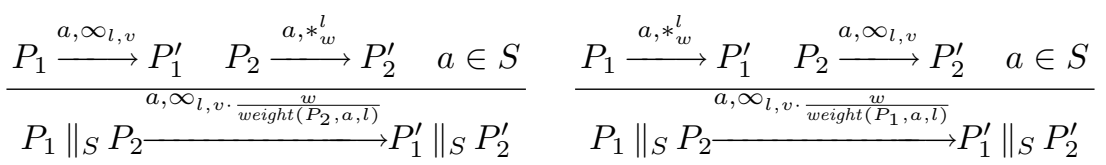

and between any two passive actions of the two terms that have the same name belonging to $S$ and the same priority constraint:

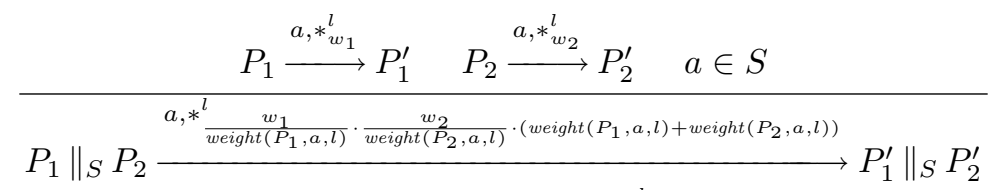

where $\operatorname{weight}(P, a, l)=\sum\left\{|w| \exists P^{\prime} \in \mathcal{P} . P \stackrel{a, *_{w}^{l}}{\longrightarrow} P^{\prime} \mid\right\}$.

The process constant $A$ behaves as the right-hand side process term in its defining equation:

$$
\frac{P \stackrel{a, \tilde{\lambda}}{\longrightarrow} P^{\prime} \quad A \triangleq P}{A \stackrel{a, \tilde{\lambda}}{\longrightarrow} P^{\prime}}
$$

From now on we use the motivating example of Sect. 1 as a running example. Let us formalize with $\mathrm{EMPA}_{\mathrm{gr}}$ the considered queueing system. The arrival process for customers of class $k \in\{1,2\}$ can be modeled as:

$$
A_{k} \triangleq<a_{k}, \lambda_{k}>A_{k}
$$

where $\lambda_{k}$ is the parameter of the Poisson arrival process. The buffer for customers of class $k \in\{1,2\}$ - which we assume to have an unbounded capacity - can be modeled as follows:

$$
\begin{aligned}
& B_{k, 0} \triangleq<a_{k}, *_{w}^{0}>\cdot B_{k, 1} \\
& B_{k, i} \triangleq<a_{k}, *_{w}^{0}>\cdot B_{k, i+1}+<d_{k}, \infty_{k, w}>\cdot B_{k, i-1} \quad i \in \mathbf{N}_{\geq 1}
\end{aligned}
$$


Each of the servers can be modeled as follows:

$$
S \triangleq<d_{1}, *{ }_{w}^{1}>. S_{1}^{\prime}+<d_{2}, *_{w}^{2}>. S_{2}^{\prime} \quad S_{1}^{\prime} \triangleq<s_{1}, \mu_{1}>. S \quad S_{2}^{\prime} \triangleq<s_{2}, \mu_{2}>. S
$$

where $\mu_{k}$ is the service rate for customers of class $k \in\{1,2\}$. Hence the overall queueing system with $n \in \mathrm{N}_{\geq 1}$ servers can be modeled as follows:

$$
Q S_{n} \triangleq\left(A_{1} \|_{\emptyset} A_{2}\right)\left\|_{\left\{a_{1}, a_{2}\right\}}\left(B_{1,0} \|_{\emptyset} B_{2,0}\right)\right\|_{\left\{d_{1}, d_{2}\right\}}\left(S\left\|_{\emptyset} S\right\|_{\emptyset} \ldots \|_{\emptyset} S\right)
$$

If we are interested in evaluating performance properties that are not specific to a single class of customers, a more abstract model can be considered like $Q S_{n} / L[\varphi]$, where $L=\left\{d_{1}, d_{2}\right\}$ and $\varphi=\left\{a_{1}, a_{2} \mapsto a, s_{1}, s_{2} \mapsto s\right\}$. The names $d_{1}, d_{2}$ of the prioritized immediate actions related to the customer delivery have been made internal, while the names $a_{1}, a_{2}$ (resp. $s_{1}, s_{2}$ ) of the exponentially timed actions related to the customer arrival (resp. service) have been mapped to the same name $a$ (resp. $s$ ). Assuming that $\lambda_{1}=\lambda_{2} \equiv \lambda$ and $\mu_{1}=\mu_{2} \equiv \mu$, we expect the performance behavior of $Q S_{n} / L[\varphi]$ to be equivalent to the performance behavior of the following simpler birth-death process:

$$
\begin{array}{ll}
B D_{n, 0} \triangleq<a, 2 \cdot \lambda>\cdot B D_{n, 1} & \\
B D_{n, j} \triangleq<a, 2 \cdot \lambda>\cdot B D_{n, j+1}+<s, j \cdot \mu>\cdot B D_{n, j-1} & 1 \leq j \leq n \\
B D_{n, j} \triangleq<a, 2 \cdot \lambda>\cdot B D_{n, j+1}+<s, n \cdot \mu>. B D_{n, j-1} & j>n
\end{array}
$$

How can we formally establish that $Q S_{n} / L[\varphi]$ is equivalent to $B D_{n, 0}$ ?

\section{Weak Extended Markovian Bisimilarity}

A behavioral equivalence typically used for reasoning about Markovian process terms is Markovian bisimilarity. ${ }^{7}$ In particular, an extension of it has been defined in Refs. 3,4 in order to deal with prioritized/weighted immediate actions. The limitation of extended Markovian bisimilarity is that it does not provide any abstraction mechanism. In fact, actions of the form $<\tau, \infty_{l, w}>$ are invisible and take no time, hence they are unimportant both from the functional viewpoint and from the performance viewpoint. Thus they should not be considered when comparing the behavior of two terms.

After recalling the definition of extended Markovian bisimilarity, we introduce a weak variant of it that abstracts from internal immediate actions. Then we show that this weak variant is a congruence and admits a sound and complete axiomatization for the class of well-prioritized process terms.

\subsection{Extended Markovian Bisimilarity}

The basic idea behind Markovian bisimilarity is to suitably compare the exit rates of the process terms. In order to deal with the various kinds of 
actions - exponentially timed, immediate, and passive - the notion of exit rate is further parameterized with respect to a number in $\mathbb{Z}$ representing the priority level of the action, which is 0 if the action is exponentially timed, $l$ if the action rate is $\infty_{l, w},-l-1$ if the action rate is $*_{w}^{l}$.

Definition 3.1. Let $P \in \mathcal{P}, a \in N a m e, l \in \mathbb{Z}$, and $C \subseteq \mathcal{P}$. The exit rate of $P$ when executing actions with name $a$ and priority level $l$ that lead to $C$ is defined through the following non-negative real function:

$$
\operatorname{rate}(P, a, l, C)= \begin{cases}\sum\left\{|\lambda| \exists P^{\prime} \in C . P \stackrel{a, \lambda}{\longrightarrow} P^{\prime} \mid\right\} & \text { if } l=0 \\ \sum\left\{|w| \exists P^{\prime} \in C . P \stackrel{a, \infty_{l, w}}{\longrightarrow} P^{\prime} \mid\right\} & \text { if } l>0 \\ \sum\left\{|w| \exists P^{\prime} \in C . P \stackrel{a, w_{w}^{-l-1}}{\longrightarrow} P^{\prime} \mid\right\} & \text { if } l<0\end{cases}
$$

where each sum is taken to be zero whenever its multiset is empty.

Extended Markovian bisimilarity compares the process term exit rates for all possible action names and priority levels, except for those actions that will always be pre-empted by higher priority actions of the form $\left\langle\tau, \infty_{l, w}\right\rangle$. In the following we denote by $\operatorname{pri}_{\infty}^{\tau}(P)$ the priority level of the highest priority internal immediate action enabled by $P$, and we set $\operatorname{pri}_{\infty}^{\tau}(P)=0$ if $P$ does not enable any internal immediate action. Moreover, given $l \in \mathbb{Z}$, we use no-pre $(l, P)$ to denote that no action whose priority level is $l$ can be pre-empted in $P$. Formally, this is the case whenever $l \geq \operatorname{pri}_{\infty}^{\tau}(P)$ or $-l-1 \geq \operatorname{pri}_{\infty}^{\tau}(P)$.

Definition 3.2. An equivalence relation $\mathcal{B} \subseteq \mathcal{P} \times \mathcal{P}$ is an extended Markovian bisimulation iff, whenever $\left(P_{1}, P_{2}\right) \in \mathcal{B}$, then for all action names $a \in$ Name, equivalence classes $C \in \mathcal{P} / \mathcal{B}$, and priority levels $l \in \mathbb{Z}$ such that no-pre $\left(l, P_{1}\right)$ and no-pre $\left(l, P_{2}\right)$ :

$$
\operatorname{rate}\left(P_{1}, a, l, C\right)=\operatorname{rate}\left(P_{2}, a, l, C\right)
$$

The definition given in Refs. 3,4 is slightly different from the one above, as the latter includes a concept of pre-emption that embodies the maximal progress of Ref. 6 . Note that, in order for $P_{1}, P_{2} \in \mathcal{P}$ to be related by an extended Markovian bisimulation, it is necessary that $\operatorname{pri}_{\infty}^{\tau}\left(P_{1}\right)=\operatorname{pri}_{\infty}^{\tau}\left(P_{2}\right)$, from which it follows that no-pre $\left(l, P_{1}\right)$ iff no-pre $\left(l, P_{2}\right)$ for all $l \in \mathbb{Z}$.

Definition 3.3. Extended Markovian bisimilarity, denoted by $\sim_{\text {EMB }}$, is the union of all the extended Markovian bisimulations.

$\sim_{\mathrm{EMB}}$ is a congruence with respect to all the operators of $\mathrm{EMPA}_{\mathrm{gr}}$. This is because the semantic rules do not prune the transitions labeled with the 
lower priority actions of a term and these are ignored by $\sim_{\mathrm{EMB}}$ only in the presence of alternative higher priority actions of the form $\left\langle\tau, \infty_{l, w}\right\rangle$ (which cannot be disabled by any context into which the term may be plugged).

$\sim_{\text {EMB }}$ admits a sound and complete axiomatization over the set $\mathcal{P}_{\text {nr }}$ of the non-recursive process terms of $\mathcal{P}$. The following six axioms characterize the race policy, the preselection policy, and pre-emption:

$$
\begin{aligned}
<a, \lambda_{1}>. P+<a, \lambda_{2}>. P & =<a, \lambda_{1}+\lambda_{2}>. P \\
<a, \infty_{l, w_{1}}>. P+<a, \infty_{l, w_{2}}>. P & =<a, \infty_{l, w_{1}+w_{2}}>. P \\
<a, *_{w_{1}}^{l}>. P+<a, *_{w_{2}}^{l}>. P & =<a, *_{w_{1}+w_{2}}^{l}>. P \\
<\tau, \infty_{l, w}>. P+<a, \lambda>. Q & =<\tau, \infty_{l, w}>. P \\
<\tau, \infty_{l, w}>. P+<a, \infty_{l^{\prime}, w^{\prime}}>. Q & =<\tau, \infty_{l, w}>. P \quad \text { if } l>l^{\prime} \\
<\tau, \infty_{l, w}>. P+<a, *_{w^{\prime}}^{l}>. Q & =<\tau, \infty_{l, w}>. P \quad \text { if } l>l^{\prime}
\end{aligned}
$$

\subsection{Abstracting from Internal Immediate Actions}

The definition of exit rate can be relaxed by means of a suitable notion of reachability that involves the unobservable actions $\left\langle\tau, \infty_{l, w}\right\rangle$. The idea is that, if a given class of process terms is not reached directly after executing an action with a certain name and priority level, then we have to explore the possibility of reaching that class indirectly via a finite-length unobservable path starting from the term reached after executing the considered action.

Definition 3.4. Let $P \in \mathcal{P}$ and $l \in \mathrm{N}_{>0}$. We say that $P$ is $l$-unobservable iff $\operatorname{pri}_{\infty}^{\tau}(P)=l$ and $P$ does not enable any visible action with priority level $l^{\prime} \in \mathbb{Z}$ such that $l^{\prime} \geq l$ or $-l^{\prime}-1 \geq l$.

Definition 3.5. Let $n \in \mathrm{N}_{>0}$ and $P_{1}, P_{2}, \ldots, P_{n+1} \in \mathcal{P}$. A path $\pi$ of length $n$ :

$$
P_{1} \stackrel{\tau, \infty_{l_{1}, w_{1}}}{\longrightarrow} P_{2} \stackrel{\tau, \infty_{l_{2}, w_{2}}}{\longrightarrow} \ldots \stackrel{\tau, \infty_{l_{n}, w_{n}}}{\longrightarrow} P_{n+1}
$$

is unobservable iff for all $i=1, \ldots, n$ process term $P_{i}$ is $l_{i}$-unobservable. In that case, the probability of executing $\pi$ is given by:

$$
\operatorname{prob}(\pi)=\prod_{i=1}^{n} \frac{w_{i}}{\operatorname{rate}\left(P_{i}, \tau, l_{i}, \mathcal{P}\right)}
$$

Definition 3.6. Let $P \in \mathcal{P}, a \in N a m e, l \in \mathbb{Z}$, and $C \subseteq \mathcal{P}$. The weak exit rate of $P$ when executing actions with name $a$ and priority level $l$ that lead to $C$ is defined through the following non-negative real function:

$$
\operatorname{rate}_{\mathrm{w}}(P, a, l, C)=\sum_{P^{\prime} \in C_{\mathrm{w}}} \operatorname{rate}\left(P, a, l,\left\{P^{\prime}\right\}\right) \cdot \operatorname{prob}_{\mathrm{w}}\left(P^{\prime}, C\right)
$$

where $C_{\mathrm{w}}$ is the weak backward closure of $C$ :

$C_{\mathrm{w}}=C \cup\{Q \in \mathcal{P}-C \mid Q$ can reach $C$ via an unobservable path $\}$ 
and prob $_{\mathrm{w}}$ is a $\mathbf{R}_{0,1}$-valued function representing the sum of the probabilities of all the unobservable paths from a term in $C_{\mathrm{w}}$ to $C$ :

$$
\operatorname{prob}_{\mathrm{w}}\left(P^{\prime}, C\right)= \begin{cases}1 & \text { if } P^{\prime} \in C \\ \sum\{|\operatorname{prob}(\pi)| \pi & \text { unobservable path from } \left.P^{\prime} \text { to } C \mid\right\} \\ & \text { if } P^{\prime} \in C_{\mathrm{w}}-C\end{cases}
$$

The definition of extended Markovian bisimulation can be weakened by using rate $_{\mathrm{w}}$ instead of rate and by skipping the weak exit rate comparison in the case of equivalence classes that are unobservable. More precisely, we distinguish between observable, initially unobservable and fully unobservable sets of states.

An observable state is a state that enables an observable action that cannot be pre-empted by any enabled unobservable action.

An initially unobservable state is a state in which all the enabled observable actions are pre-empted by some enabled unobservable action, but at least one of the paths starting at this state with one of the higher priority enabled unobservable actions reaches an observable state.

A fully unobservable state is a state in which all the enabled observable actions are pre-empted by some enabled unobservable action, and all the paths starting at this state with one of the higher priority enabled unobservable actions are unobservable (note that $\underline{0}$ is fully unobservable).

The weak exit rate comparison must obviously be performed in the case of observable classes, while it is not necessary in the case of initially unobservable classes as each of them can reach an observable class. Besides being not necessary, the weak exit rate comparison with respect to initially unobservable classes may even be counterproductive in terms of abstraction power, as we shall exemplify later on. Thus, the weak exit rate comparison must be skipped in the case of initially unobservable classes. By contrast, the weak exit rate comparison should not be skipped in the case of fully unobservable classes. This is especially true for process terms that conceptually do not behave the same but can reach only fully unobservable states, as in this case the only way we have to distinguish between those process terms is to compare their weak exit rates towards such fully unobservable states. As we shall exemplify later on, in the weak exit rate comparison it is not appropriate to consider the fully unobservable classes one at a time. Instead, the whole set $\mathcal{P}_{\text {fu }}$ of fully unobservable process terms of $\mathcal{P}$ has to be considered.

Definition 3.7. An equivalence relation $\mathcal{B} \subseteq \mathcal{P} \times \mathcal{P}$ is a weak extended Markovian bisimulation iff, whenever $\left(P_{1}, P_{2}\right) \in \mathcal{B}$, then for all action 
names $a \in N a m e$ and priority levels $l \in \mathbb{Z}$ such that no-pre $\left(l, P_{1}\right)$ and no-pre $\left(l, P_{2}\right)$ :

$$
\begin{aligned}
\operatorname{rate}_{\mathrm{w}}\left(P_{1}, a, l, C\right) & =\operatorname{rate}_{\mathrm{w}}\left(P_{2}, a, l, C\right) \text { for all observable } C \in \mathcal{P} / \mathcal{B} \\
\operatorname{rate}_{\mathrm{w}}\left(P_{1}, a, l, \mathcal{P}_{\mathrm{fu}}\right) & =\operatorname{rate}_{\mathrm{w}}\left(P_{2}, a, l, \mathcal{P}_{\mathrm{fu}}\right)
\end{aligned}
$$

Definition 3.8. Weak extended Markovian bisimilarity, denoted by $\approx_{\mathrm{EMB}}$, is the union of all the weak extended Markovian bisimulations.

Example 3.1. Consider the two following process terms:

$$
\begin{aligned}
& P_{1} \equiv<a, \lambda>.<\tau, \infty_{l, w}>.<b, \mu>. \underline{0} \\
& P_{2} \equiv<a, \lambda>.<b, \mu>. \underline{0}
\end{aligned}
$$

Then $P_{1} \approx_{\text {EMB }} P_{2}$. In fact, denoted by $I d_{\mathcal{P}}$ the identity relation over $\mathcal{P}$, the equivalence relation $\mathcal{B}_{1,2}=\left\{\left(P_{1}, P_{2}\right),\left(P_{2}, P_{1}\right)\right\} \cup I d_{\mathcal{P}}$ turns out to be a weak extended Markovian bisimulation. In particular: rate $_{\mathrm{W}}\left(P_{1}, a, 0,[<b, \mu>. \underline{0}]_{\mathcal{B}_{1,2}}\right)=\lambda \cdot \frac{w}{w}=\lambda \cdot 1=\operatorname{rate}_{\mathrm{w}}\left(P_{2}, a, 0,[<b, \mu>. \underline{0}]_{\mathcal{B}_{1,2}}\right)$ Note that the equivalence class $\left[\left\langle\tau, \infty_{l, w}\right\rangle .\langle b, \mu\rangle . \underline{0}\right]_{\mathcal{B}_{1,2}}$ has not been considered in the rate $_{\mathrm{w}}$-based comparison as it is initially unobservable. If it had been considered, then we would have obtained:

$$
\begin{aligned}
& \text { rate }_{\mathrm{w}}\left(P_{1}, a, 0,\left[\left\langle\tau, \infty_{l, w}>.<b, \mu>. \underline{0}\right]_{\mathcal{B}_{1,2}}\right)=\lambda \cdot 1=\lambda\right. \\
& \text { rate }_{\mathrm{w}}\left(P_{2}, a, 0,\left[<\tau, \infty_{l, w}>.<b, \mu>. \underline{0}\right]_{\mathcal{B}_{1,2}}\right)=0
\end{aligned}
$$

which would have led us to the counterintuitive conclusion that $P_{1}$ and $P_{2}$ are not weakly extended Markovian bisimilar.

Example 3.2. Consider the two following process terms:

$$
\begin{aligned}
& P_{3} \equiv<a, \lambda>.\left(<\tau, \infty_{l, w_{1}}>.<b, \mu>. \underline{0}+<\tau, \infty_{l, w_{2}}>.<c, \gamma>. \underline{0}\right) \\
& P_{4} \equiv<a, \lambda \cdot \frac{w_{1}}{w_{1}+w_{2}}>\cdot<b, \mu>. \underline{0}+<a, \lambda \cdot \frac{w_{2}}{w_{1}+w_{2}}>.<c, \gamma>. \underline{0}
\end{aligned}
$$

Then $P_{3} \approx_{\text {EMB }} P_{4}$. Indeed, $\mathcal{B}_{3,4}=\left\{\left(P_{3}, P_{4}\right),\left(P_{4}, P_{3}\right)\right\} \cup I d_{\mathcal{P}}$ turns out to be a weak extended Markovian bisimulation. In particular:

$$
\begin{aligned}
\operatorname{rate}_{\mathrm{w}}\left(P_{3}, a, 0,[<b, \mu>. \underline{0}]_{\mathcal{B}_{3,4}}\right)=\lambda \cdot \frac{w_{1}}{w_{1}+w_{2}} & =\left(\lambda \cdot \frac{w_{1}}{w_{1}+w_{2}}\right) \cdot 1= \\
& =\operatorname{rate}_{\mathrm{w}}\left(P_{4}, a, 0,[<b, \mu>. \underline{0}]_{\mathcal{B}_{3,4}}\right) \\
\operatorname{rate}_{\mathrm{w}}\left(P_{3}, a, 0,[<c, \gamma>. \underline{0}]_{\mathcal{B}_{3,4}}\right)=\lambda \cdot \frac{w_{2}}{w_{1}+w_{2}} & =\left(\lambda \cdot \frac{w_{2}}{w_{1}+w_{2}}\right) \cdot 1= \\
& =\operatorname{rate}_{\mathrm{w}}\left(P_{4}, a, 0,[<c, \gamma>. \underline{0}]_{\mathcal{B}_{3,4}}\right)
\end{aligned}
$$

Note that $P_{3}$ and $P_{4}$ are also weakly extended Markovian bisimilar to:

$$
\begin{aligned}
P_{3}^{\prime} & \equiv<a, \lambda>. A \\
A & \triangleq<\tau, \infty_{l, w_{1}}>.<b, \mu>. \underline{0}+<\tau, \infty_{l, w_{2}}>.<c, \gamma>. \underline{0}+<\tau, \infty_{l, w_{3}}>. A
\end{aligned}
$$

In fact, denoted by $W$ the sum $w_{1}+w_{2}+w_{3}$, we have:

$$
\begin{aligned}
\text { rate }_{\mathrm{w}}\left(P_{3}^{\prime}, a, 0,[<b, \mu>. \underline{0}]_{\mathcal{B}_{3,4}}\right) & =\lambda \cdot \sum_{i=0}^{\infty}\left(\frac{w_{3}}{W}\right)^{i} \cdot \frac{w_{1}}{W}=\lambda \cdot \frac{w_{1}}{W} \cdot \frac{1}{1-w_{3} / W}= \\
& =\lambda \cdot \frac{w_{1}}{W} \cdot \frac{W}{W-w_{3}}=\lambda \cdot \frac{w_{1}}{w_{1}+w_{2}} \\
\text { rate }_{\mathrm{w}}\left(P_{3}^{\prime}, a, 0,[<c, \gamma>. \underline{0}]_{\mathcal{B}_{3,4}}\right) & =\lambda \cdot \frac{w_{2}}{w_{1}+w_{2}}
\end{aligned}
$$


This means that $\approx_{\mathrm{EMB}}$ can abstract not only from intermediate unobservable actions (see Ex. 3.1) but also from intermediate unobservable self-loops, consistently with the fact that the probability to escape from them is 1 .

Example 3.3. Consider the two following process terms:

$$
\begin{aligned}
& P_{5} \equiv<\tau, \infty_{l, w}>.<a, \lambda>. \underline{0} \\
& P_{6} \equiv<a, \lambda>. \underline{0}
\end{aligned}
$$

Although in principle one may view $P_{5}$ and $P_{6}$ as being equivalent, they are not related by $\approx_{\mathrm{EMB}}$. The reason is that $P_{5}$ can reach $P_{6}$ after executing an immediate $\tau$-action while $P_{6}$ cannot reach itself after executing any action. However, the fact that $P_{5} \varkappa_{\mathrm{EMB}} P_{6}$ avoids a congruence violation. It is well known that any weak bisimulation equivalence relating a process term that can execute an initial unobservable action with one that cannot breaks compositionality with respect to the alternative composition operator. ${ }^{9}$

Example 3.4. Consider the two following process terms:

$$
\begin{aligned}
& P_{7} \equiv<a_{1}, \lambda_{1}>\cdot A_{1}, \quad A_{1} \triangleq<\tau, \infty_{l_{1}, w_{1}}>\cdot A_{1} \\
& P_{8} \equiv<a_{2}, \lambda_{2}>\cdot A_{2}, \quad A_{2} \triangleq<\tau, \infty_{l_{2}, w_{2}}>\cdot A_{2}
\end{aligned}
$$

where $\left.\left\langle a_{1}, \lambda_{1}\right\rangle \neq<a_{2}, \lambda_{2}\right\rangle$. Then $P_{7} \not z_{\text {EMB }} P_{8}$. In fact, observed that $A_{1}, A_{2} \in \mathcal{P}_{\text {fu }}$, from $<a_{1}, \lambda_{1}>\neq<a_{2}, \lambda_{2}>$ it follows that for $a_{1} \neq a_{2}$ :

$$
\text { rate }_{\mathrm{w}}\left(P_{7}, a_{1}, 0, \mathcal{P}_{\mathrm{fu}}\right)=\lambda_{1} \cdot 1 \neq 0=\operatorname{rate}_{\mathrm{w}}\left(P_{8}, a_{1}, 0, \mathcal{P}_{\mathrm{fu}}\right)
$$

while for $a_{1}=a_{2}$ :

$$
\text { rate }_{\mathrm{w}}\left(P_{7}, a_{1}, 0, \mathcal{P}_{\mathrm{fu}}\right)=\lambda_{1} \cdot 1 \neq \lambda_{2} \cdot 1=\operatorname{rate}_{\mathrm{w}}\left(P_{8}, a_{1}, 0, \mathcal{P}_{\mathrm{fu}}\right)
$$

If we had not considered $\mathcal{P}_{\mathrm{fu}}$ in Def. 3.7, then there would have been no way to distinguish between $P_{7}$ and $P_{8}$, as they cannot reach any observable state.

Example 3.5. Consider the two following process terms:

$$
\begin{aligned}
P_{9} & \equiv<a, \lambda>. A_{1}, \quad A_{1} \triangleq<\tau, \infty_{l_{1}, w_{1}}>\cdot A_{1} \\
P_{10} & \equiv<a, \lambda>. A_{2}, \quad A_{2} \triangleq<\tau, \infty_{l_{2}, w_{2}}>\cdot A_{2}
\end{aligned}
$$

where $A_{1}$ and $A_{2}$ are defined as in the previous example. Then $P_{9} \approx_{\mathrm{EMB}}$ $P_{10}$. In fact, the equivalence relation $\mathcal{B}_{9,10}=\left\{\left(P_{9}, P_{10}\right),\left(P_{10}, P_{9}\right)\right\} \cup I d_{\mathcal{P}}$ turns out to be a weak extended Markovian bisimulation. In particular, recalled that $A_{1}, A_{2} \in \mathcal{P}_{\text {fu }}$, it holds that:

$$
\text { rate }_{\mathrm{w}}\left(P_{9}, a, 0, \mathcal{P}_{\mathrm{fu}}\right)=\lambda \cdot 1=\operatorname{rate}_{\mathrm{w}}\left(P_{10}, a, 0, \mathcal{P}_{\mathrm{fu}}\right)
$$

Note that $A_{1} \not_{\mathrm{EMB}} A_{2}$ whenever $\left.<\tau, \infty_{l_{1}, w_{1}}\right\rangle \neq<\tau, \infty_{l_{2}, w_{2}}>$. Thus, in this case, if we had considered the two fully unobservable classes $\left[A_{1}\right]_{\mathcal{B}_{9,10}}$ and $\left[A_{2}\right]_{\mathcal{B}_{9,10}}$ separately, then we would have obtained: 


$$
\begin{aligned}
& \text { rate }_{\mathrm{w}}\left(P_{9}, a, 0,\left[A_{1}\right]_{\mathcal{B}_{9,10}}\right)=\lambda \cdot 1 \neq 0=\text { rate }_{\mathrm{w}}\left(P_{10}, a, 0,\left[A_{1}\right]_{\mathcal{B}_{9,10}}\right) \\
& \text { rate }_{\mathrm{w}}\left(P_{9}, a, 0,\left[A_{2}\right]_{\mathcal{B}_{9,10}}\right)=0 \neq \lambda \cdot 1=\text { rate }_{\mathrm{w}}\left(P_{10}, a, 0,\left[A_{2}\right]_{\mathcal{B}_{9,10}}\right)
\end{aligned}
$$

which would have led us to the counterintuitive conclusion that $P_{9}$ and $P_{10}$ are not weakly extended Markovian bisimilar.

We point out that $P_{9}$ and $P_{10}$ are also weakly extended Markovian bisimilar to $P_{6} \equiv\left\langle a, \lambda>. \underline{0}\right.$ as from $\underline{0} \in \mathcal{P}_{\text {fu }}$ it follows:

$$
\text { rate }_{\mathrm{w}}\left(P_{6}, a, 0, \mathcal{P}_{\mathrm{fu}}\right)=\lambda \cdot 1
$$

Hence $\approx_{\text {EMB }}$ can abstract not only from intermediate unobservable selfloops (see Ex. 3.2) but also from terminal unobservable self-loops, consistently with the fact that from them it is not possible to escape at all.

Going back to our running example formalized in Sect. 2, it is easy to see that $Q S_{n} / L[\varphi] \approx_{\mathrm{EMB}} B D_{n, 0}$. For instance, if we consider the case of $n=2$ servers, the relation $\mathcal{B}_{Q S_{2} / L[\varphi], B D_{2,0}}$ given by $I d_{\mathcal{P}}$ plus the following pairs and their symmetrical ones:

$$
\begin{aligned}
& \left(\left(\left(A_{1} \|_{\emptyset} A_{2}\right)\left\|_{\left\{a_{1}, a_{2}\right\}}\left(B_{1,0} \|_{\emptyset} B_{2,0}\right)\right\|_{\left\{d_{1}, d_{2}\right\}}\left(S \|_{\emptyset} S\right)\right) / L[\varphi], \quad B D_{2,0}\right) \\
& \left(\left(\left(A_{1} \|_{\emptyset} A_{2}\right)\left\|_{\left\{a_{1}, a_{2}\right\}}\left(B_{1,0} \|_{\emptyset} B_{2,0}\right)\right\|_{\left\{d_{1}, d_{2}\right\}}\left(S_{l}^{\prime} \|_{\emptyset} S\right)\right) / L[\varphi], \quad B D_{2,1}\right) \\
& \left(\left(\left(A_{1} \|_{\emptyset} A_{2}\right)\left\|_{\left\{a_{1}, a_{2}\right\}}\left(B_{1,0} \|_{\emptyset} B_{2,0}\right)\right\|_{\left\{d_{1}, d_{2}\right\}}\left(S \|_{\emptyset} S_{l}^{\prime}\right)\right) / L[\varphi], \quad B D_{2,1}\right) \\
& \left(\left(\left(A_{1} \|_{\emptyset} A_{2}\right)\left\|_{\left\{a_{1}, a_{2}\right\}}\left(B_{1, i_{1}} \|_{\emptyset} B_{2, i_{2}}\right)\right\|_{\left\{d_{1}, d_{2}\right\}}\left(S_{h}^{\prime} \|_{\emptyset} S_{k}^{\prime}\right)\right) / L[\varphi], \quad B D_{2, j}\right)
\end{aligned}
$$
turns out to be a weak extended Markovian bisimulation whenever $l, h, k \in$ $\{1,2\}$ and $i_{1}, i_{2} \in \mathbf{N}, j \in \mathbf{N}_{\geq 2}$ are such that $i_{1}+i_{2}=j-2$.

\subsection{Congruence Result for Well-Prioritized Terms}

We now investigate the congruence property for $\approx_{\text {EMB }}$. We start with the operators of EMPAgr different from parallel composition.

Theorem 3.1. Let $P_{1}, P_{2} \in \mathcal{P}$. Whenever $P_{1} \approx_{\mathrm{EMB}} P_{2}$, then:

(1) $<a, \tilde{\lambda}>$. $P_{1} \approx_{\mathrm{EMB}}<a, \tilde{\lambda}>$. $P_{2}$ for all $<a, \tilde{\lambda}>\in$ Act.

(2) $P_{1}+P \approx_{\mathrm{EMB}} P_{2}+P$ and $P+P_{1} \approx_{\mathrm{EMB}} P+P_{2}$ for all $P \in \mathcal{P}$.

(3) $P_{1} / L \approx_{\mathrm{EMB}} P_{2} / L$ for all $L \subseteq$ Name $-\{\tau\}$.

(4) $P_{1}[\varphi] \approx_{\mathrm{EMB}} P_{2}[\varphi]$ for all $\varphi \in$ Relab.

When applying the alternative composition operator or the parallel composition operator to two process terms, it may happen that an unobservable state of one term is combined with an observable state of the other term. Given $P_{1}, P_{2} \in \mathcal{P}$ such that $P_{1} \approx_{\text {EMB }} P_{2}$, these hybrid state combinations raise no congruence violation in the case of the alternative compositions $P_{1}+P$ and $P_{2}+P$ for any $P \in \mathcal{P}$. The reason is that the only combined 
states are the initial states of the two compositions. Such initial states include the initial states of $P_{1}$ and $P_{2}$, which must agree on the value of any weak exit rate considered in Def. 3.7.

Unfortunately, problems do arise in the parallel composition case. The first problem is concerned with initially (resp. fully) unobservable states different from the initial one that are combined with observable states, as the comparison of the weak exit rates towards the classes containing such states can no longer be skipped (resp. included into $\mathcal{P}_{\text {fu }}$ ). Consider e.g.:

$$
\begin{aligned}
P_{1} & \equiv<a, \lambda>.<\tau, \infty_{l_{1}, w_{1}}>.<b, \mu>. \underline{0} \\
P_{2} & \equiv<a, \lambda>.<\tau, \infty_{l_{2}, w_{2}}>.<b, \mu>. \underline{0} \\
P & \equiv<a, *_{w}^{0}>.<c, \infty_{l^{\prime}, w^{\prime}}>. \underline{0}
\end{aligned}
$$

with $\infty_{l_{1}, w_{1}} \neq \infty_{l_{2}, w_{2}}$. It is easy to see that $P_{1} \approx_{\text {EMB }} P_{2}$. Although the following two states of $P_{1}$ and $P_{2}$ are initially unobservable:

$$
\begin{aligned}
& <\tau, \infty_{l_{1}, w_{1}}>.<b, \mu>. \underline{0} \\
& <\tau, \infty_{l_{2}, w_{2}}>.<b, \mu>. \underline{0}
\end{aligned}
$$

in $P_{1} \|_{\{a\}} P$ and $P_{2} \|_{\{a\}} P$ the two corresponding states:

$$
\begin{aligned}
& <\tau, \infty_{l_{1}, w_{1}}>.<b, \mu>. \underline{0} \|_{\{a\}}<c, \infty_{l^{\prime}, w^{\prime}}>. \underline{0} \\
& <\tau, \infty_{l_{2}, w_{2}}>.<b, \mu>. \underline{0} \|_{\{a\}}<c, \infty_{l^{\prime}, w^{\prime}}>. \underline{0}
\end{aligned}
$$

are observable whenever $c \neq \tau$ and $l^{\prime} \geq \max \left(l_{1}, l_{2}\right)$. From $\infty_{l_{1}, w_{1}} \neq \infty_{l_{2}, w_{2}}$ it follows that the two corresponding observable states are not weakly extended Markovian bisimilar, so we can conclude that $P_{1} \|_{\{a\}} P \not_{\mathrm{EMB}}$ $P_{2} \|_{\{a\}} P$ as each of them can only reach one of the two new observable states. The same congruence violation would happen in the case that the $c$-action were passive. A similar violation - in which only one of the two initially unobservable states becomes observable - would happen for $c \neq \tau$ and $\min \left(l_{1}, l_{2}\right) \leq l^{\prime}<\max \left(l_{1}, l_{2}\right)$. Analogous violations would take place in the absence of action $\langle b, \mu\rangle$ from $P_{1}$ and $P_{2}$, which would make the two considered original states fully unobservable.

The second congruence-related problem with parallel composition is due to the fact that a different pre-emption scheme may be imposed by unobservable actions of the same process term to initially or fully unobservable states (different from the initial one) of two weakly extended Markovian bisimilar terms. A congruence violation of this kind would happen in the example above in the case that $P$ were replaced by:

$$
P^{\prime} \equiv<a, *_{w}^{0}>\cdot<\tau, \infty_{l^{\prime}, w^{\prime}}>\cdot<c, \infty_{l^{\prime}, w^{\prime}}>. \underline{0}
$$

with $l_{1}<l_{2}=l^{\prime}$ and $c \neq \tau$. In fact, after executing $<a, \lambda>$, only $P_{2} \|_{\{a\}} P^{\prime}$ would reach via an unobservable path the only observable state in which both $\langle b, \mu\rangle$ and $\left\langle c, \infty_{l^{\prime}, w^{\prime}}\right\rangle$ are enabled. An analogous violation would take place in the absence of action $\left\langle b, \mu>\right.$ from $P_{1}$ and $P_{2}$. 
In order to overcome these two congruence-related problems, given $P_{1} \|_{S} P$ and $P_{2} \|_{S} P$ with $P_{1} \approx_{\text {EMB }} P_{2}$, we have to restrict ourselves to contexts $P$ that preserve the unobservable states of $\llbracket P_{1} \rrbracket$ and $\llbracket P_{2} \rrbracket$ as well as the pre-emption schemes of their transitions.

The third congruence-related problem with parallel composition is concerned with terminal states - which are fully unobservable states with no outgoing transitions, like $\underline{0}$ - and unescapable paths - which are infinite paths traversing only fully unobservable states. It may happen that a process term with terminal states and a process term with unescapable paths are weakly extended Markovian bisimilar but can be distinguished when placed in parallel with the same process term. This is the case e.g. of terms $P_{9}$ and $P_{6}$ considered in Ex. 3.5. They are weakly extended Markovian bisimilar, but if we place each of them in the context of - $\left.\|_{\{a\}}<a, *_{w}^{0}\right\rangle .<c, \gamma>$. $\underline{0}$, after executing $\langle a, \lambda\rangle$ the first composition gets stuck into an unobservable self-loop while the second composition reaches an observable state in which $\langle c, \gamma\rangle$ is enabled.

Theorem 3.2. Let $P_{1}, P_{2} \in \mathcal{P}$ such that both $\llbracket P_{1} \rrbracket$ and $\llbracket P_{2} \rrbracket$ have no unescapable paths or no terminal states. Whenever $P_{1} \approx_{\mathrm{EMB}} P_{2}$, then $P_{1}\left\|_{S} P \approx_{\mathrm{EMB}} P_{2}\right\|_{S} P$ and $P\left\|_{S} P_{1} \approx_{\mathrm{EMB}} P\right\|_{S} P_{2}$ for all $S \subseteq$ Name $-\{\tau\}$ and $P \in \mathcal{P}$ such that any immediate/passive transition of $\llbracket P \rrbracket$ has priority level/constraint less than the priority level of any unobservable transition departing from an unobservable state of $\llbracket P_{1} \rrbracket$ or $\llbracket P_{2} \rrbracket$.

Definition 3.9. The set $\mathcal{P}_{\mathrm{wp}}$ of the well-prioritized process terms is the smallest subset of $\mathcal{P}$ closed with respect to null term, action prefix, alternative composition, hiding, relabeling, recursion and closed with respect to parallel composition in the following sense: If $P_{1}, P_{2} \in \mathcal{P}_{\text {wp }}$ and any immediate/passive transition of each of $\llbracket P_{1} \rrbracket$ and $\llbracket P_{2} \rrbracket$ has priority level/constraint less than the priority level of any unobservable transition departing from an unobservable state of the other one, then $P_{1} \|_{S} P_{2} \in \mathcal{P}_{\text {wp }}$.

Corollary 3.1. $\approx_{\mathrm{EMB}}$ is a congruence with respect to all the operators over the set $\mathcal{P}_{\mathrm{wp}}^{\prime}$ (resp. $\left.\mathcal{P}_{\mathrm{wp}}^{\prime \prime}\right)$ of the well-prioritized process terms without unescapable paths (resp. terminal states).

\subsection{Axioms for Non-Recursive Well-Prioritized Terms}

The equational laws characterizing $\approx_{\mathrm{EMB}}$ over the set $\mathcal{P}_{\mathrm{wp}, \mathrm{nr}}$ of the nonrecursive process terms of $\mathcal{P}_{\text {wp }}$ are described by the set $\mathcal{A}$ of axioms shown in Tables 1 and 2 . These are the same axioms as for $\sim_{\text {EMB }}$ with in addition the 
Table 1. Axiomatization of $\approx_{\mathrm{EMB}}$ over $\mathcal{P}_{\mathrm{wp}, \mathrm{nr}}($ part I)

\begin{tabular}{|c|c|c|}
\hline $\begin{array}{l}\left(\mathcal{A}_{1}\right) \\
\left(\mathcal{A}_{2}\right) \\
\left(\mathcal{A}_{3}\right) \\
\left(\mathcal{A}_{4}\right) \\
\left(\mathcal{A}_{5}\right) \\
\left(\mathcal{A}_{6}\right) \\
\left(\mathcal{A}_{7}\right) \\
\left(\mathcal{A}_{8}\right) \\
\left(\mathcal{A}_{9}\right) \\
\left(\mathcal{A}_{10}\right)\end{array}$ & 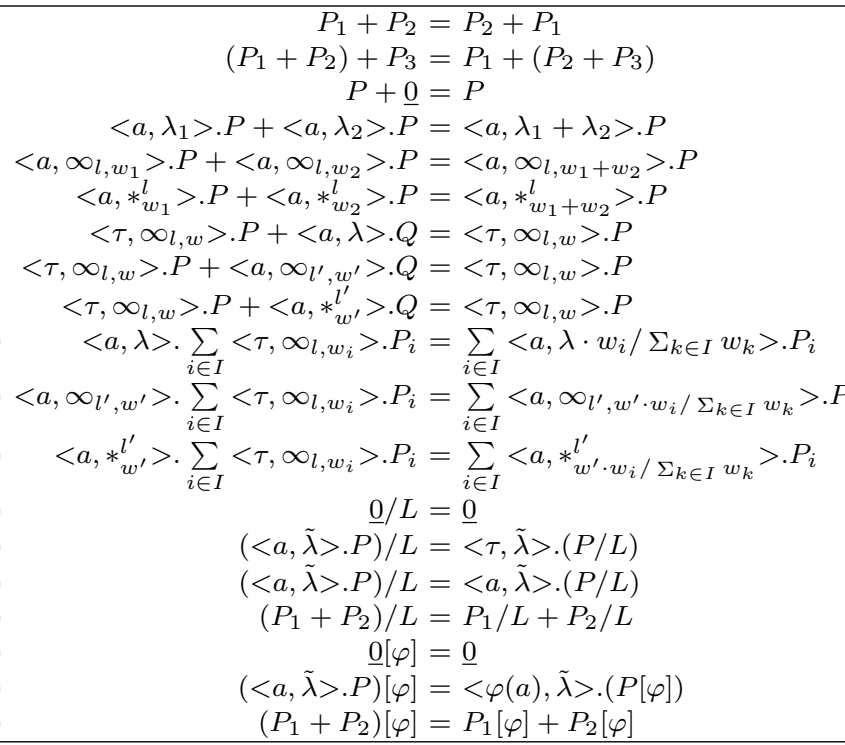 & $\begin{array}{l}\text { if } a \in L \\
\text { if } a \notin L\end{array}$ \\
\hline
\end{tabular}

Table 2. Axiomatization of $\approx_{\mathrm{EMB}}$ over $\mathcal{P}_{\mathrm{wp}, \mathrm{nr}}$ (part II - expansion law)

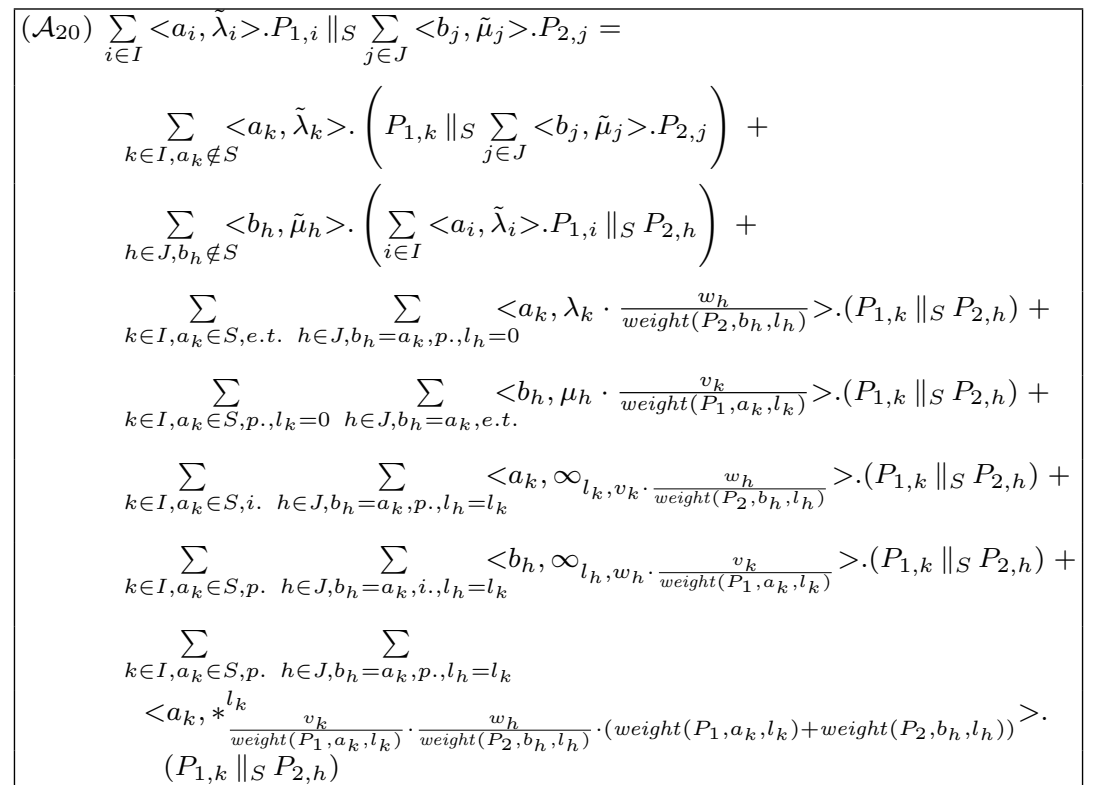


three axiom schemata $\mathcal{A}_{10}-\mathcal{A}_{12}$, in each of which the index set $I$ is finite and non-empty. Such axiom schemata express the capability of abstracting from non-initial immediate $\tau$-actions, provided that the weights of these actions are taken into account when setting the rates of the copies of the actions preceding them. As far as the expansion law $\mathcal{A}_{20}$ for parallel composition is concerned, $I$ and $J$ are finite index sets (if empty, the related summations are taken to be $\underline{0}$ ), "e.t." stands for exponentially timed, "i." stands for immediate, and "p." stands for passive. We observe that $\mathcal{P}_{\text {wp,nr }} \subseteq \mathcal{P}_{\text {wp }}^{\prime}$ as its process terms are not recursive (hence no unescapable paths).

Theorem 3.3. $D E D(\mathcal{A})$ is sound and complete for $\approx_{\mathrm{EMB}}$ over $\mathcal{P}_{\mathrm{wp}, \mathrm{nr}}$ :

$$
\mathcal{A} \vdash P_{1}=P_{2} \Longleftrightarrow P_{1} \approx_{\mathrm{EMB}} P_{2} \quad \text { for all } P_{1}, P_{2} \in \mathcal{P}_{\mathrm{wp}, \mathrm{nr}}
$$

\section{Conclusion}

Building on Refs. 6,10 in this paper we have defined a weak variant of Markovian bisimilarity, which abstracts from prioritized/weighted actions that are invisible and take zero time. This allows us to cope with expressive calculi like EMPA $\mathrm{gr}_{\mathrm{gr}}$, in which GSPN-like immediate actions are firstclass citizens that freely alternate with exponentially timed actions. On the technical side, besides carefully classifying the states on the basis of their functional and performance observability, we have introduced priority constraints for passive actions together with the class of well-prioritized process terms in order to achieve congruence.

The restriction of compositionality to well-prioritized process terms is an intrinsic limit of $\approx_{\mathrm{EMB}}$, which has nothing to do with the way in which priorities and weights are dealt with. In EMPA $\mathrm{gr}_{\mathrm{gr}}$ these are associated locally with the immediate actions of the individual components, but can also be used globally to choose among immediate actions enabled in different components. This is the source of a modeling problem known as confusion in the GSPN field, which can be solved by setting the priority levels of the immediate actions in a way that avoids interference among components. ${ }^{11}$

On the other hand, separation of concerns can be achieved by considering the priorities and weights associated with the immediate actions of the individual components to be strictly local and - in a way inspired by Ref. 2 - by assigning global priorities and weights to the occurrences of the parallel composition operator, which thus becomes ${ }^{L_{1}, W_{1}} \|_{S}{ }^{L_{2}, W_{2}}$ and preselects which component is allowed to perform immediate actions. However, even in such a setting the congruence problems with parallel composition mentioned in Sect. 3.3 would persist. As an example, consider the process 
terms $P_{1}$ and $P_{2}$ of Ex. 3.1, which are weakly extended Markovian bisimilar, and the process term $P \equiv\left\langle a, *_{w^{\prime}}^{0}>.<c, \infty_{l^{\prime}, w^{\prime}}>\right.$. 0 , where $c \neq \tau$. Then $P_{1}{ }^{L_{1}, W_{1}}\left\|_{\{a\}}{ }^{L_{2}, W_{2}} P \not_{\mathrm{EMB}} P_{2}{ }^{L_{1}, W_{1}}\right\|_{\{a\}}{ }^{L_{2}, W_{2}} P$ for $L_{1}=L_{2}$ because, after synchronizing on $a$, the first composed term enables a $\tau$-action and a $c$-action while the second composed term enables a $b$-action and a $c$-action.

The restriction to well-prioritized process terms is not a severe limitation in practice. Compositionality and abstraction of $\approx_{\mathrm{EMB}}$ can in fact be exploited if the parallel subterms composing an arbitrary process term are considered from the most prioritized one to the least prioritized one and the application of the hiding operators is anticipated/postponed accordingly. Consider for instance the following process term:

$$
\left(\ldots\left(\left(\left(\left(P_{1} \|_{S_{1-2}} P_{2}\right) / H_{1-2}\right) \|_{S_{1-3}} P_{3}\right) / H_{1-3}\right) \ldots \|_{S_{1-n}} P_{n}\right) / H_{1-n}
$$

If we call priority of a process term $P$ the maximum $l \in \mathbb{Z}$ such that no-pre $(l, P)$, then the subterms $P_{1}, P_{2}, \ldots, P_{n}$ can be considered in this order if the priority of $P_{i}$ is greater than the priority of $P_{i+1}$ for all $i=$ $1, \ldots, n-1$, otherwise a permutation of the subterms is necessary (together with the adjustment of the synchronization sets and hiding sets). This will be the subject of future work.

\section{References}

1. M. Ajmone Marsan, G. Balbo, G. Conte, S. Donatelli, and G. Franceschinis, Modelling with Generalized Stochastic Petri Nets (J. Wiley \& Sons, 1995).

2. J.C.M. Baeten, J.A. Bergstra, and S.A. Smolka, Axiomatizing Probabilistic Processes: ACP with Generative Probabilities, in Information and Computation 121:234-255 (1995).

3. M. Bernardo, Theory and Application of Extended Markovian Process Algebra, Ph.D. Thesis (University of Bologna, Italy, 1999).

4. M. Bernardo and M. Bravetti, Performance Measure Sensitive Congruences for Markovian Process Algebras, in Theoretical Computer Science 290:117160 (2003).

5. R.J. van Glabbeek, S.A. Smolka, and B. Steffen, Reactive, Generative and Stratified Models of Probabilistic Processes, in Information and Computation 121:59-80 (1995).

6. H. Hermanns, Interactive Markov Chains, LNCS 2428 (2002).

7. J. Hillston, A Compositional Approach to Performance Modelling (Cambridge University Press, 1996).

8. L. Kleinrock, Queueing Systems (J. Wiley \& Sons, 1975).

9. R. Milner, Communication and Concurrency (Prentice Hall, 1989).

10. M. Rettelbach, Probabilistic Branching in Markovian Process Algebras, in Computer Journal 38:590-599 (1995).

11. E. Teruel, G. Franceschinis, and M. De Pierro, Well-Defined Generalized Stochastic Petri Nets: A Net-Level Method to Specify Priorities, in IEEE Trans. on Software Engineering 29:962-973 (2003). 


\section{Appendix A. Proofs}

First of all we establish the well-foundedness of the definition of $\approx_{\text {EMB }}$.

Lemma A.1. Let $I$ be a non-empty set and $\mathcal{B}_{i} \subseteq \mathcal{P} \times \mathcal{P}$ be a weak extended Markovian bisimulation for all $i \in I$. Then $\mathcal{B}=\left(\cup_{i \in I} \mathcal{B}_{i}\right)^{+}$is a weak extended Markovian bisimulation.

Proof. Observed that $\mathcal{B}$ is an equivalence relation because it is the transitive closure of the union of equivalence relations, we can redefine it as $\mathcal{B}=\cup_{n \in \mathbb{N}_{>0}} \mathcal{B}^{(n)}$ where $\mathcal{B}^{(n)}=\left(\cup_{i \in I} \mathcal{B}_{i}\right)^{n}$. Thus $\left(P_{1}, P_{2}\right) \in \mathcal{B}$ iff $\left(P_{1}, P_{2}\right) \in \mathcal{B}^{(n)}$ for some $n \in \mathbf{N}_{>0}$. As a consequence, the result follows if we prove that, whenever $\left(P_{1}, P_{2}\right) \in \mathcal{B}^{(n)}$ for some $n \in \mathbf{N}_{>0}$, then for all $a \in N a m e$ and $l \in \mathbb{Z}$ such that no-pre $\left(l, P_{1}\right)$ and no-pre $\left(l, P_{2}\right)$ :

$$
\begin{aligned}
\operatorname{rate}_{\mathrm{w}}\left(P_{1}, a, l, C\right) & =\text { rate }_{\mathrm{w}}\left(P_{2}, a, l, C\right) \quad \text { for all observable } C \in \mathcal{P} / \mathcal{B} \\
\text { rate }_{\mathrm{w}}\left(P_{1}, a, l, \mathcal{P}_{\mathrm{fu}}\right) & =\operatorname{rate}_{\mathrm{w}}\left(P_{2}, a, l, \mathcal{P}_{\mathrm{fu}}\right)
\end{aligned}
$$

Let us proceed by induction on $n \in \mathrm{N}_{>0}$ :

- If $n=1$ then $\left(P_{1}, P_{2}\right) \in \mathcal{B}_{i}$ for some $i \in I$. Since $\mathcal{B}_{i}$ is a weak extended Markovian bisimulation, we immediately derive:

$$
\operatorname{rate}_{\mathrm{w}}\left(P_{1}, a, l, \mathcal{P}_{\mathrm{fu}}\right)=\operatorname{rate}_{\mathrm{w}}\left(P_{2}, a, l, \mathcal{P}_{\mathrm{fu}}\right)
$$

Let $\mathcal{P} / \mathcal{B}_{i}=\left\{C_{i, j} \mid j \in J_{i}\right\}$. Since $\mathcal{B}_{i} \subseteq \mathcal{B}$, for all $C_{i, j} \in \mathcal{P} / \mathcal{B}_{i}$ there exists $C \in \mathcal{P} / \mathcal{B}$ such that $C_{i, j} \subseteq C$. Thus, every (observable) equivalence class of $\mathcal{B}$ can be written as the union of a number of (observable) equivalence classes of $\mathcal{B}_{i}$. As a consequence, for all observable $C=\cup_{j \in J_{i}^{\prime}} C_{i, j} \in \mathcal{P} / \mathcal{B}$ where $J_{i}^{\prime} \subseteq J_{i}$, it holds for each $i=1,2$ :

$$
\begin{aligned}
\operatorname{rate~}_{\mathrm{w}}\left(P_{i}, a, l, C\right) & =\sum_{P^{\prime} \in C_{\mathrm{w}}} \operatorname{rate}\left(P_{i}, a, l,\left\{P^{\prime}\right\}\right) \cdot \operatorname{prob}_{\mathrm{w}}\left(P^{\prime}, C\right)= \\
& =\sum_{P^{\prime} \in C_{\mathrm{w}}}\left(\operatorname{rate}\left(P_{i}, a, l,\left\{P^{\prime}\right\}\right) \cdot \sum_{j \in J_{i}^{\prime}} \operatorname{prob}_{\mathrm{w}}\left(P^{\prime}, C_{i, j}\right)\right)= \\
& =\sum_{j \in J_{i}^{\prime}} \sum_{P^{\prime} \in C_{i, j, \mathrm{w}}} \operatorname{rate}\left(P_{i}, a, l,\left\{P^{\prime}\right\}\right) \cdot \operatorname{prob}_{\mathrm{w}}\left(P^{\prime}, C_{i, j}\right)= \\
& =\sum_{j \in J_{i}^{\prime}} \operatorname{rate}_{\mathrm{w}}\left(P_{i}, a, l, C_{i, j}\right)
\end{aligned}
$$

Since $\mathcal{B}_{i}$ is a weak extended Markovian bisimulation, for all $j \in J_{i}^{\prime}$ :

hence:

$$
\operatorname{rate}_{\mathrm{w}}\left(P_{1}, a, l, C_{i, j}\right)=\operatorname{rate}_{\mathrm{w}}\left(P_{2}, a, l, C_{i, j}\right)
$$

$$
\text { rate }_{\mathrm{w}}\left(P_{1}, a, l, C\right)=\operatorname{rate}_{\mathrm{w}}\left(P_{2}, a, l, C\right)
$$


- If $n>1$ from $\left(P_{1}, P_{2}\right) \in \mathcal{B}^{(n)}$ we derive that there exists $Q \in \mathcal{P}$ such that $\left(P_{1}, Q\right) \in \mathcal{B}^{(n-1)}$ and $\left(Q, P_{2}\right) \in \mathcal{B}_{i}$ for some $i \in I$. From the induction hypothesis it follows that:

$$
\begin{aligned}
\text { rate }_{\mathrm{w}}\left(P_{1}, a, l, C\right) & =\text { rate }_{\mathrm{w}}(Q, a, l, C) \quad \text { for all observable } C \in \mathcal{P} / \mathcal{B} \\
\text { rate }_{\mathrm{w}}\left(P_{1}, a, l, \mathcal{P}_{\mathrm{fu}}\right) & =\text { rate }_{\mathrm{w}}\left(Q, a, l, \mathcal{P}_{\mathrm{fu}}\right)
\end{aligned}
$$

From what we proved in the case $n=1$ it follows that:

$$
\begin{aligned}
\text { rate }_{\mathrm{w}}(Q, a, l, C) & =\text { rate }_{\mathrm{w}}\left(P_{2}, a, l, C\right) \quad \text { for all observable } C \in \mathcal{P} / \mathcal{B} \\
\text { rate }_{\mathrm{w}}\left(Q, a, l, \mathcal{P}_{\mathrm{fu}}\right) & =\text { rate }_{\mathrm{w}}\left(P_{2}, a, l, \mathcal{P}_{\mathrm{fu}}\right)
\end{aligned}
$$

As a consequence, the result follows by transitivity.

Lemma A.2. $\approx_{\mathrm{EMB}}$ is the largest weak extended Markovian bisimulation.

Proof. Since $\approx_{\mathrm{EMB}}$ is the union of all the weak extended Markovian bisimulations, it will contain in particular the largest weak extended Markovian bisimulation. So, if we prove that $\approx_{\mathrm{EMB}}$ is a weak extended Markovian bisimulation, then we are done.

We first observe that $\approx_{\mathrm{EMB}}=\approx_{\mathrm{EMB}}^{+}$. In fact, $\approx_{\mathrm{EMB}} \subseteq \approx_{\mathrm{EMB}}^{+}$trivially holds. On the other hand, $\approx_{\mathrm{EMB}}^{+} \subseteq \approx_{\mathrm{EMB}}$ is due to the fact that $\approx_{\mathrm{EMB}}^{+}$is a weak extended Markovian bisimulation (by virtue of Lemma A.1) and that $\approx_{\mathrm{EMB}}$ contains all the weak extended Markovian bisimulations (by definition). From $\approx_{\mathrm{EMB}}=\approx_{\mathrm{EMB}}^{+}$it follows that $\approx_{\mathrm{EMB}}$ is a weak extended Markovian bisimulation because so is $\approx_{\mathrm{EMB}}^{+}$(by virtue of Lemma A.1).

Then we present some properties that will be useful later on to prove the congruence result as well as the soundness and the completeness of the axiomatization for $\approx_{\mathrm{EMB}}$.

Lemma A.3. Let $P \in \mathcal{P}, a \in N a m e$, and $l \in \mathbb{Z}$. Then:

$$
\text { rate }_{\mathrm{w}}(P, a, l, \mathcal{P})=\operatorname{rate}(P, a, l, \mathcal{P})
$$

Proof. Since $\mathcal{P}_{\mathrm{w}}=\mathcal{P}$, we immediately derive:

$$
\begin{aligned}
\text { rate }_{\mathrm{w}}(P, a, l, \mathcal{P}) & =\sum_{P^{\prime} \in \mathcal{P}_{\mathrm{w}}} \operatorname{rate}\left(P, a, l,\left\{P^{\prime}\right\}\right) \cdot \operatorname{prob}_{\mathrm{w}}\left(P^{\prime}, \mathcal{P}\right) \\
& =\sum_{P^{\prime} \in \mathcal{P}} \operatorname{rate}\left(P, a, l,\left\{P^{\prime}\right\}\right) \cdot 1 \\
& =\operatorname{rate}(P, a, l, \mathcal{P})
\end{aligned}
$$


Lemma A.4. Let $P \in \mathcal{P}, a \in$ Name, $l \in \mathbb{Z}$, and $C \subseteq \mathcal{P}$. Then:

$$
\operatorname{rate}(P, a, l, C) \leq \operatorname{rate}_{\mathrm{w}}(P, a, l, C) \leq \operatorname{rate}(P, a, l, \mathcal{P})
$$

Proof. The first inequality stems from the fact that, besides all the atransitions of priority $l$ from $P$ to $C$, there might be other a-transitions of priority $l$ departing from $P$ that do not reach $C$ in one step but lead to unobservable states from which $C$ can be reached via unobservable paths. The second inequality derives from rate ${ }_{\mathrm{w}}(P, a, l, C) \leq$ rate $_{\mathrm{w}}(P, a, l, \mathcal{P})$ and Lemma A.3.

Lemma A.5. Let $P_{1}, P_{2} \in \mathcal{P}$. Whenever $P_{1} \sim_{\mathrm{EMB}} P_{2}$, then $P_{1} \approx_{\mathrm{EMB}} P_{2}$.

Proof. We prove that any extended Markovian bisimulation $\mathcal{B}$ including $\left(P_{1}, P_{2}\right)$ is a weak extended Markovian bisimulation too, from which the result will follow.

Let $\left(Q_{1}, Q_{2}\right) \in \mathcal{B}, a \in$ Name, $C \in \mathcal{P} / \mathcal{B}$, and $l \in \mathbb{Z}$ such that no-pre $\left(l, Q_{1}\right)$ and no-pre $\left(l, Q_{2}\right)$.

From Lemma A.4, $\left(Q_{1}, Q_{2}\right) \in \mathcal{B}$, and the fact that $\mathcal{B}$ is an extended Markovian bisimulation, it follows that:

$\operatorname{rate}_{\mathrm{w}}\left(Q_{1}, a, l, C\right) \geq \operatorname{rate}\left(Q_{1}, a, l, C\right)=\operatorname{rate}\left(Q_{2}, a, l, C\right) \leq \operatorname{rate}_{\mathrm{w}}\left(Q_{2}, a, l, C\right)$ Since $\left(Q_{1}, Q_{2}\right) \in \mathcal{B}$ and $\mathcal{B}$ is an extended Markovian bisimulation, both $Q_{1}$ and $Q_{2}$ or neither of them enable a-actions of priority $l$ that do not reach $C$ in one step but lead to unobservable states from which $C$ can be reached via unobservable paths.

If neither of them does, then we immediately get:

$\operatorname{rate}_{\mathrm{w}}\left(Q_{1}, a, l, C\right)=\operatorname{rate}\left(Q_{1}, a, l, C\right)=\operatorname{rate}\left(Q_{2}, a, l, C\right)=\operatorname{rate}_{\mathrm{w}}\left(Q_{2}, a, l, C\right)$ If instead both of them do, consider the following aggregate unobservable path traversing $C^{(1)}, C^{(2)}, \ldots, C^{(n)} \in \mathcal{P} / \mathcal{B}$, where $Q_{1}$ reaches $C^{(1)}$ after executing an a-action of priority $l, C^{(n)}=C$, and $P^{(i)} \in C^{(i)}$ is $l^{(i)}$ unobservable for $i=1, \ldots, n-1$ :

$$
C^{(1)} \stackrel{\tau, \operatorname{rate}\left(P^{(1)}, \tau, l^{(1)}, C^{(2)}\right)}{\longrightarrow} \ldots \stackrel{\tau, \operatorname{rate}\left(P^{(n-1)}, \tau, l^{(n-1)}, C^{(n)}\right)}{\longrightarrow} C^{(n)}
$$

This aggregate path contributes to rate ${ }_{\mathrm{w}}\left(Q_{1}, a, l, C\right)-$ rate $\left(Q_{1}, a, l, C\right)$ with:

$$
\operatorname{rate}\left(Q_{1}, a, l, C^{(1)}\right) \cdot \prod_{i=1}^{n-1} \frac{\operatorname{rate}\left(P^{(i)}, \tau, l^{(i)}, C^{(i+1)}\right)}{\operatorname{rate}\left(P^{(i)}, \tau, l^{(i)}, \mathcal{P}\right)}
$$

Since $\left(Q_{1}, Q_{2}\right) \in \mathcal{B}, C^{(1)}, C^{(2)}, \ldots, C^{(n)} \in \mathcal{P} / \mathcal{B}$, and $\mathcal{B}$ is an extended 
Markovian bisimulation, $Q_{2}$ must have an aggregate unobservable path that traverses the same equivalence classes and brings the same contribution to rate $_{\mathrm{w}}\left(Q_{2}, a, l, C\right)-\operatorname{rate}\left(Q_{2}, a, l, C\right)$.

From the generality of the considered aggregate path it follows that:

rate $_{\mathrm{w}}\left(Q_{1}, a, l, C\right)-\operatorname{rate}\left(Q_{1}, a, l, C\right)=\operatorname{rate}_{\mathrm{w}}\left(Q_{2}, a, l, C\right)-\operatorname{rate}\left(Q_{2}, a, l, C\right)$ hence from $\left(Q_{1}, Q_{2}\right) \in \mathcal{B}$ with $\mathcal{B}$ being an extended Markovian bisimulation we derive that:

$$
\text { rate }_{\mathrm{w}}\left(Q_{1}, a, l, C\right)=\text { rate }_{\mathrm{w}}\left(Q_{2}, a, l, C\right)
$$

From the generality of $C$ it follows that the first clause of Def. 3.7 is satisfied.

As far as the second clause of Def. 3.7 is concerned, it suffices to observe that for each $i=1,2$ :

$$
\text { rate }_{\mathrm{w}}\left(Q_{i}, a, l, \mathcal{P}_{\mathrm{fu}}\right)=\sum_{C \in \mathcal{P} / \mathcal{B} \text { fully unobservable }} \text { rate }_{\mathrm{w}}\left(Q_{i}, a, l, C\right)
$$

Lemma A.6. Let $P_{1}, P_{2} \in \mathcal{P}$. Whenever $P_{1} \approx_{\mathrm{EMB}} P_{2}$, then for all $a \in N a m e$ and $l \in \mathbb{Z}$ such that no-pre $\left(l, P_{1}\right)$ and no-pre $\left(l, P_{2}\right)$ :

$$
\text { rate }_{\mathrm{w}}\left(P_{1}, a, l, \mathcal{P}\right)=\text { rate }_{\mathrm{w}}\left(P_{2}, a, l, \mathcal{P}\right)
$$

Proof. It suffices to observe that for each $i=1,2$ :

$$
\text { rate }_{\mathrm{w}}\left(P_{i}, a, l, \mathcal{P}\right)=\sum_{C \in \mathcal{P} / \approx_{\mathrm{EMB}} \text { observable }} \text { rate }_{\mathrm{W}}\left(P_{i}, a, l, C\right)+\text { rate }_{\mathrm{w}}\left(P_{i}, a, l, \mathcal{P}_{\mathrm{fu}}\right)
$$

Lemma A.7. Let $P_{1}, P_{2} \in \mathcal{P}$. Whenever $P_{1} \approx_{\mathrm{EMB}} P_{2}$, then for all $a \in N a m e$ and $l \in \mathbb{Z}$ such that no-pre $\left(l, P_{1}\right)$ and no-pre $\left(l, P_{2}\right)$ :

$$
\operatorname{rate}\left(P_{1}, a, l, \mathcal{P}\right)=\operatorname{rate}\left(P_{2}, a, l, \mathcal{P}\right)
$$

Proof. From Lemma A.3 and Lemma A.6 we immediately derive:

$$
\operatorname{rate}\left(P_{1}, a, l, \mathcal{P}\right)=\operatorname{rate}_{\mathrm{w}}\left(P_{1}, a, l, \mathcal{P}\right)=\operatorname{rate}_{\mathrm{w}}\left(P_{2}, a, l, \mathcal{P}\right)=\operatorname{rate}\left(P_{2}, a, l, \mathcal{P}\right)
$$

Lemma A.8. Let $C \subseteq \mathcal{P}$ and $P \in C_{\mathrm{w}}-C$ be l-unobservable. Then:

$$
\operatorname{prob}_{\mathrm{w}}(P, C)=\operatorname{rate}_{\mathrm{w}}(P, \tau, l, C) / \operatorname{rate}(P, \tau, l, \mathcal{P})
$$

Proof. Recalled that: 


$$
\operatorname{rate}_{\mathrm{w}}(P, \tau, l, C)=\sum_{P^{\prime} \in C_{\mathrm{w}}} \operatorname{rate}\left(P, \tau, l,\left\{P^{\prime}\right\}\right) \cdot \operatorname{prob}_{\mathrm{w}}\left(P^{\prime}, C\right)
$$

in order to move from rate ${ }_{\mathrm{w}}(P, \tau, l, C)$ to $\operatorname{prob}_{\mathrm{w}}(P, C)$ all we have to do is to consider the probability (instead of the rate) of the first $\tau$-transition of each unobservable path from $P$ to $C$. This is obtained by dividing the rate of that transition by rate $(P, \tau, l, \mathcal{P})$.

Finally we prove the results stated in the paper.

Proof of Thm. 3.1. Let $\mathcal{B}$ be a weak extended Markovian bisimulation such that $\left(P_{1}, P_{2}\right) \in \mathcal{B}$.

(1) Given $<a, \tilde{\lambda}>\in$ Act of priority level $l$, we prove that the transitive closure $\mathcal{B}^{\prime}$ of:

$$
\mathcal{B} \cup\left\{\left(<a, \tilde{\lambda}>. P_{1},<a, \tilde{\lambda}>. P_{2}\right),\left(<a, \tilde{\lambda}>\cdot P_{2},<a, \tilde{\lambda}>. P_{1}\right)\right\}
$$

is a weak extended Markovian bisimulation. Note that any element of $\mathcal{P} / \mathcal{B}^{\prime}$ either coincides with an element of $\mathcal{P} / \mathcal{B}$ or is $\left.[<a, \tilde{\lambda}\rangle . P_{1}\right]_{\mathcal{B}} \cup$ $\left[<a, \tilde{\lambda}>. P_{2}\right]_{\mathcal{B}}$, so we can concentrate on the rate $_{\mathrm{w}}$-based comparison for $<a, \tilde{\lambda}>. P_{1}$ and $<a, \tilde{\lambda}>. P_{2}$ only.

There are three cases:

- If $P_{1}$ and $P_{2}$ are observable, then the only class reachable by $<a, \tilde{\lambda}>\cdot P_{1}$ and $\left\langle a, \tilde{\lambda}>. P_{2}\right.$ is $\left[P_{1}\right]_{\mathcal{B}^{\prime}}$, which includes $\left[P_{1}\right]_{\mathcal{B}}$ hence both $P_{1}$ and $P_{2}$. For each $i=1,2$ we have:

$$
\operatorname{rate}_{\mathrm{w}}\left(<a, \tilde{\lambda}>. P_{i}, a, l,\left[P_{1}\right]_{\mathcal{B}^{\prime}}\right)=\operatorname{rate}\left(<a, \tilde{\lambda}>. P_{i}, a, l,\left[P_{1}\right]_{\mathcal{B}^{\prime}}\right)
$$

where:

$$
\operatorname{rate}\left(<a, \tilde{\lambda}>. P_{1}, a, l,\left[P_{1}\right]_{\mathcal{B}^{\prime}}\right)=\operatorname{rate}\left(<a, \tilde{\lambda}>. P_{2}, a, l,\left[P_{1}\right]_{\mathcal{B}^{\prime}}\right)
$$

Since the weak exit rate of both terms is zero in all the other cases, the result follows.

- If $P_{1}$ and $P_{2}$ are initially $l^{\prime}$-unobservable, by virtue of Lemma A.8 we have for each $i=1,2$ :

$$
\begin{aligned}
\operatorname{rate}_{\mathrm{w}}\left(<a, \tilde{\lambda}>. P_{i}, a, l, C\right) & =\operatorname{rate}\left(<a, \tilde{\lambda}>. P_{i}, a, l, P_{i}\right) \cdot \operatorname{prob}_{\mathrm{w}}\left(P_{i}, C\right)= \\
& =\operatorname{rate}\left(<a, \tilde{\lambda}>. P_{i}, a, l, P_{i}\right) \cdot \frac{\operatorname{rate}_{\mathrm{w}}\left(P_{i}, \tau, l^{\prime}, C\right)}{\operatorname{rate}\left(P_{i}, \tau, l^{\prime}, \mathcal{P}\right)}
\end{aligned}
$$

where:

$$
\operatorname{rate}\left(<a, \tilde{\lambda}>. P_{1}, a, l, P_{1}\right)=\operatorname{rate}\left(<a, \tilde{\lambda}>. P_{2}, a, l, P_{2}\right)
$$

and (by virtue of $\left(P_{1}, P_{2}\right) \in \mathcal{B}$ with $\mathcal{B}$ weak extended Markovian 
bisimulation and Lemma A.7):

$$
\begin{aligned}
\operatorname{rate}_{\mathrm{w}}\left(P_{1}, \tau, l^{\prime}, C\right) & =\operatorname{rate}_{\mathrm{w}}\left(P_{2}, \tau, l^{\prime}, C\right) \\
\operatorname{rate}\left(P_{1}, \tau, l^{\prime}, \mathcal{P}\right) & =\operatorname{rate}\left(P_{2}, \tau, l^{\prime}, \mathcal{P}\right)
\end{aligned}
$$

for all $C \in \mathcal{P} / \mathcal{B}^{\prime}$ observable (due to the relationship between the classes of $\mathcal{B}^{\prime}$ and the classes of $\mathcal{B}$ ) as well as for $C=\mathcal{P}_{\text {fu }}$. Since the weak exit rate of both terms is zero in all the other cases, the result follows.

- If $P_{1}$ and $P_{2}$ are fully unobservable, then for each $i=1,2$ we have:

$$
\text { rate }_{\mathrm{w}}\left(<a, \tilde{\lambda}>. P_{i}, a, l, \mathcal{P}_{\mathrm{fu}}\right)=\operatorname{rate}\left(<a, \tilde{\lambda}>. P_{i}, a, l, P_{i}\right)
$$

where:

$$
\operatorname{rate}\left(<a, \tilde{\lambda}>. P_{1}, a, l, P_{1}\right)=\operatorname{rate}\left(<a, \tilde{\lambda}>. P_{2}, a, l, P_{2}\right)
$$

Since the weak exit rate of both terms is zero in all the other cases, the result follows.

(2) Given $P \in \mathcal{P}$, we prove that the transitive closure $\mathcal{B}^{\prime}$ of:

$$
\mathcal{B} \cup\left\{\left(P_{1}+P, P_{2}+P\right),\left(P_{2}+P, P_{1}+P\right)\right\}
$$

is a weak extended Markovian bisimulation. Note that any element of $\mathcal{P} / \mathcal{B}^{\prime}$ either coincides with an element of $\mathcal{P} / \mathcal{B}$ or is $\left[P_{1}+P\right]_{\mathcal{B}} \cup\left[P_{2}+P\right]_{\mathcal{B}}$, so we can concentrate on the rate $_{\mathrm{w}}$-based comparison for $P_{1}+P$ and $P_{2}+P$ only.

Let $a \in N a m e$ and $l \in \mathbb{Z}$ such that no-pre $\left(l, P_{1}+P\right)$ and no-pre $\left(l, P_{2}+\right.$ $P)$. For each $i=1,2$ we have:

$$
\begin{aligned}
\text { rate }_{\mathrm{w}}\left(P_{i}+P, a, l, C\right) & =\sum_{P^{\prime} \in C_{\mathrm{w}}} \operatorname{rate}\left(P_{i}+P, a, l,\left\{P^{\prime}\right\}\right) \cdot \operatorname{prob}_{\mathrm{w}}\left(P^{\prime}, C\right)= \\
& =\sum_{P^{\prime} \in C_{\mathrm{w}}} \operatorname{rate}\left(P_{i}, a, l,\left\{P^{\prime}\right\}\right) \cdot \operatorname{prob}_{\mathrm{w}}\left(P^{\prime}, C\right)+ \\
& \sum_{P^{\prime} \in C_{\mathrm{w}}} \operatorname{rate}\left(P, a, l,\left\{P^{\prime}\right\}\right) \cdot \operatorname{prob}_{\mathrm{w}}\left(P^{\prime}, C\right)= \\
& \operatorname{rate}_{\mathrm{w}}\left(P_{i}, a, l, C\right)+\operatorname{rate}_{\mathrm{w}}(P, a, l, C)
\end{aligned}
$$

where (by virtue of $\left(P_{1}, P_{2}\right) \in \mathcal{B}$ with $\mathcal{B}$ weak extended Markovian bisimulation):

$$
\text { rate }_{\mathrm{w}}\left(P_{1}, a, l, C\right)=\text { rate }_{\mathrm{w}}\left(P_{2}, a, l, C\right)
$$

for all $C \in \mathcal{P} / \mathcal{B}^{\prime}$ observable (due to the relationship between the classes of $\mathcal{B}^{\prime}$ and the classes of $\mathcal{B}$ ) as well as for $C=\mathcal{P}_{\text {fu }}$. The result then follows.

(3) Given $L \subseteq$ Name $-\{\tau\}$, we prove that:

$$
\mathcal{B}^{\prime}=\left\{\left(Q_{1} / L, Q_{2} / L\right) \mid\left(Q_{1}, Q_{2}\right) \in \mathcal{B}\right\} \cup I d_{\mathcal{P}}
$$

is a weak extended Markovian bisimulation. Note that any element of 
$\mathcal{P} / \mathcal{B}^{\prime}$ is either $[Q / L]_{\mathcal{B}^{\prime}}=[Q]_{\mathcal{B}} / L$ or $[R]_{\mathcal{B}^{\prime}}=\{R\}$ with the outermost operator of $R$ different from -/L, so we can concentrate on the rate $_{\mathrm{w}^{-}}$ based comparison for process terms of the form $Q / L$ only.

Let $\left(Q_{1} / L, Q_{2} / L\right) \in \mathcal{B}^{\prime}, a \in$ Name, and $l \in \mathbb{Z}$ such that no-pre $\left(l, Q_{1} / L\right)$ and no-pre $\left(l, Q_{2} / L\right)$. Observed that the weak exit rate of both $Q_{1} / L$ and $Q_{2} / L$ towards classes of the form $[R]_{\mathcal{B}^{\prime}}$ with the outermost operator of $R$ different from ${ }_{-} / L$ is zero, we further let $C=[Q / L]_{\mathcal{B}^{\prime}}$ observable.

For each $i=1,2$ we have:

rate $_{\mathrm{w}}\left(Q_{i} / L, a, l, C\right)=\left\{\begin{array}{cc}\operatorname{rate}_{\mathrm{w}}\left(Q_{i}, a, l,[Q]_{\mathcal{B}}\right)+a d d_{\mathrm{w}}\left(Q_{i}, a, l, C, L\right) \\ 0 \quad & \text { if } a \in N a m e-(L \cup\{\tau\}) \\ 0 & \text { if } a \in L \\ \sum_{b \in L \cup\{\tau\}}\left(\text { rate }_{\mathrm{w}}\left(Q_{i}, b, l,[Q]_{\mathcal{B}}\right)+a d d_{\mathrm{w}}\left(Q_{i}, b, l, C, L\right)\right) & \text { if } a=\tau\end{array}\right.$

where for all $c \in$ Name:

$$
a d d_{\mathrm{w}}\left(Q_{i}, c, l, C, L\right)=\sum_{\left[Q^{\prime}\right]_{\mathcal{B}} \text { obs. s.t. }\left[Q^{\prime} / L\right]_{\mathcal{B}^{\prime}} \text { unobs. }} \operatorname{rate}_{\mathrm{w}}\left(Q_{i}, c, l,\left[Q^{\prime}\right]_{\mathcal{B}}\right) \cdot \operatorname{prob}_{\mathrm{w}}\left(Q^{\prime} / L, C\right)
$$

is the additional weak exit rate due to observable classes that become unobservable when applying $-/ L$.

From $\left(Q_{1} / L, Q_{2} / L\right) \in \mathcal{B}^{\prime}$ it follows that $\left(Q_{1}, Q_{2}\right) \in \mathcal{B}$. Since $\mathcal{B}$ is a weak extended Markovian bisimulation, for all $c \in$ Name we have that:

and:

$$
\text { rate }_{\mathrm{w}}\left(Q_{1}, c, l,[Q]_{\mathcal{B}}\right)=\text { rate }_{\mathrm{w}}\left(Q_{2}, c, l,[Q]_{\mathcal{B}}\right)
$$

$$
\text { rate }_{\mathrm{w}}\left(Q_{1}, c, l,\left[Q^{\prime}\right]_{\mathcal{B}}\right)=\text { rate }_{\mathrm{w}}\left(Q_{2}, c, l,\left[Q^{\prime}\right]_{\mathcal{B}}\right)
$$

for any observable class $\left[Q^{\prime}\right]_{\mathcal{B}}$ such that $\left[Q^{\prime} / L\right]_{\mathcal{B}^{\prime}}$ is unobservable, from which the first part of the result follows.

The case $C=\mathcal{P}_{\mathrm{fu}}$ can be treated similarly to the previous one after replacing every occurrence of $[Q]_{\mathcal{B}}$ with $\mathcal{P}_{\mathrm{fu}}$.

(4) Given $\varphi \in$ Relab, we prove that:

$$
\mathcal{B}^{\prime}=\left\{\left(Q_{1}[\varphi], Q_{2}[\varphi]\right) \mid\left(Q_{1}, Q_{2}\right) \in \mathcal{B}\right\} \cup I d_{\mathcal{P}}
$$

is a weak extended Markovian bisimulation. Note that any element of $\mathcal{P} / \mathcal{B}^{\prime}$ is either $[Q[\varphi]]_{\mathcal{B}^{\prime}}=[Q]_{\mathcal{B}}[\varphi]$ or $[R]_{\mathcal{B}^{\prime}}=\{R\}$ with the outermost operator of $R$ different from ${ }_{-}[\varphi]$, so we can concentrate on the rate $_{\mathrm{w}^{-}}$ based comparison for process terms of the form $Q[\varphi]$ only.

Let $\left(Q_{1}[\varphi], Q_{2}[\varphi]\right) \in \mathcal{B}^{\prime}, a \in$ Name, and $l \in \mathbb{Z}$ such that 
no-pre $\left(l, Q_{1}[\varphi]\right)$ and no-pre $\left(l, Q_{2}[\varphi]\right)$. Observed that the weak exit rate of both $Q_{1}[\varphi]$ and $Q_{2}[\varphi]$ towards classes of the form $[R]_{\mathcal{B}^{\prime}}$ with the outermost operator of $R$ different from $-[\varphi]$ is zero, we further let $C=[Q[\varphi]]_{\mathcal{B}^{\prime}}$ observable.

For each $i=1,2$ we have:

$$
\text { rate }_{\mathrm{w}}\left(Q_{i}[\varphi], a, l, C\right)=\sum_{b \in \varphi^{-1}(a)} \text { rate }_{\mathrm{w}}\left(Q_{i}, b, l,[Q]_{\mathcal{B}}\right)
$$

From $\left(Q_{1}[\varphi], Q_{2}[\varphi]\right) \in \mathcal{B}^{\prime}$ it follows that $\left(Q_{1}, Q_{2}\right) \in \mathcal{B}$. Since $\mathcal{B}$ is a weak extended Markovian bisimulation, for all $b \in \varphi^{-1}(a)$ we have that:

$$
\text { rate }_{\mathrm{w}}\left(Q_{1}, b, l,[Q]_{\mathcal{B}}\right)=\text { rate }_{\mathrm{w}}\left(Q_{2}, b, l,[Q]_{\mathcal{B}}\right)
$$

from which the first part of the result follows.

The case $C=\mathcal{P}_{\mathrm{fu}}$ can be treated similarly to the previous one after replacing every occurrence of $[Q]_{\mathcal{B}}$ with $\mathcal{P}_{\text {fu }}$.

Proof of Thm. 3.2. Let $\mathcal{B}$ be a weak extended Markovian bisimulation over $\mathcal{P}$ such that $\left(P_{1}, P_{2}\right) \in \mathcal{B}$. Given $S \subseteq$ Name $-\{\tau\}$, we prove that:

$\mathcal{B}^{\prime}=\left\{\left(Q_{1}\left\|_{S} Q, Q_{2}\right\|_{S} Q\right) \mid\left(Q_{1}, Q_{2}\right) \in \mathcal{B}\right.$ and $Q_{1}, Q_{2}, Q$ satisf. the hyp. $\} \cup I d_{\mathcal{P}}$ is a weak extended Markovian bisimulation over $\mathcal{P}$, where $Q_{1}, Q_{2}, Q$ satisfying the hypothesis means that both $Q_{1}$ and $Q_{2}$ have no unescapable paths or no terminal states and that any immediate/passive transition of $\llbracket Q \rrbracket$ has priority level/constraint less than the priority level of any unobservable transition departing from an unobservable state of $\llbracket Q_{1} \rrbracket$ or $\llbracket Q_{2} \rrbracket$. Note that any element of $\mathcal{P} / \mathcal{B}^{\prime}$ is either $\left[Q^{\prime} \|_{S} Q^{\prime \prime}\right]_{\mathcal{B}^{\prime}}=\left[Q^{\prime}\right]_{\mathcal{B}} \|_{S} Q^{\prime \prime}$ with all the process terms in $\left[Q^{\prime}\right]_{\mathcal{B}}$ having no unescapable paths or no terminal states and $Q^{\prime \prime}$ satisfying the priority constraint, or $[R]_{\mathcal{B}^{\prime}}=\{R\}$ with the outermost operator of $R$ different from $\|_{S}$. Thus we can concentrate on the rate $_{\mathrm{w}}$-based comparison for process terms of the form $Q^{\prime} \|_{S} Q^{\prime \prime}$ only. Let $\left(Q_{1}\left\|_{S} Q, Q_{2}\right\|_{S} Q\right) \in \mathcal{B}^{\prime}, a \in$ Name, and $l \in \mathbb{Z}$ such that no-pre $\left(l, Q_{1} \|_{S} Q\right)$ and no-pre $\left(l, Q_{2} \|_{S} Q\right)$. Observed that the weak exit rate of both $Q_{1} \|_{S} Q$ and $Q_{2} \|_{S} Q$ towards classes of the form $[R]_{\mathcal{B}^{\prime}}$ with the outermost operator of $R$ different from ${ }_{-} \|_{S}$ - is zero, we further let $C=\left[Q^{\prime} \|_{S} Q^{\prime \prime}\right]_{\mathcal{B}^{\prime}}$ observable.

Since $Q_{1}, Q_{2}, Q$ satisfy the hypothesis, the unobservable states of $Q_{1}$ and $Q_{2}$ remain unobservable in $Q_{1} \|_{S} Q$ and $Q_{2} \|_{S} Q$, respectively. As a consequence, the unobservable paths of $Q_{1}$ and $Q_{2}$ are inherited by $Q_{1} \|_{S} Q$ and $Q_{2} \|_{S} Q$, respectively, unless they are preceded by transitions that are pruned because of synchronization constraints. 
Thus for each $i=1,2$ we have that rate $_{\mathrm{w}}\left(Q_{i} \|_{S} Q, a, l, C\right)$ is equal to:

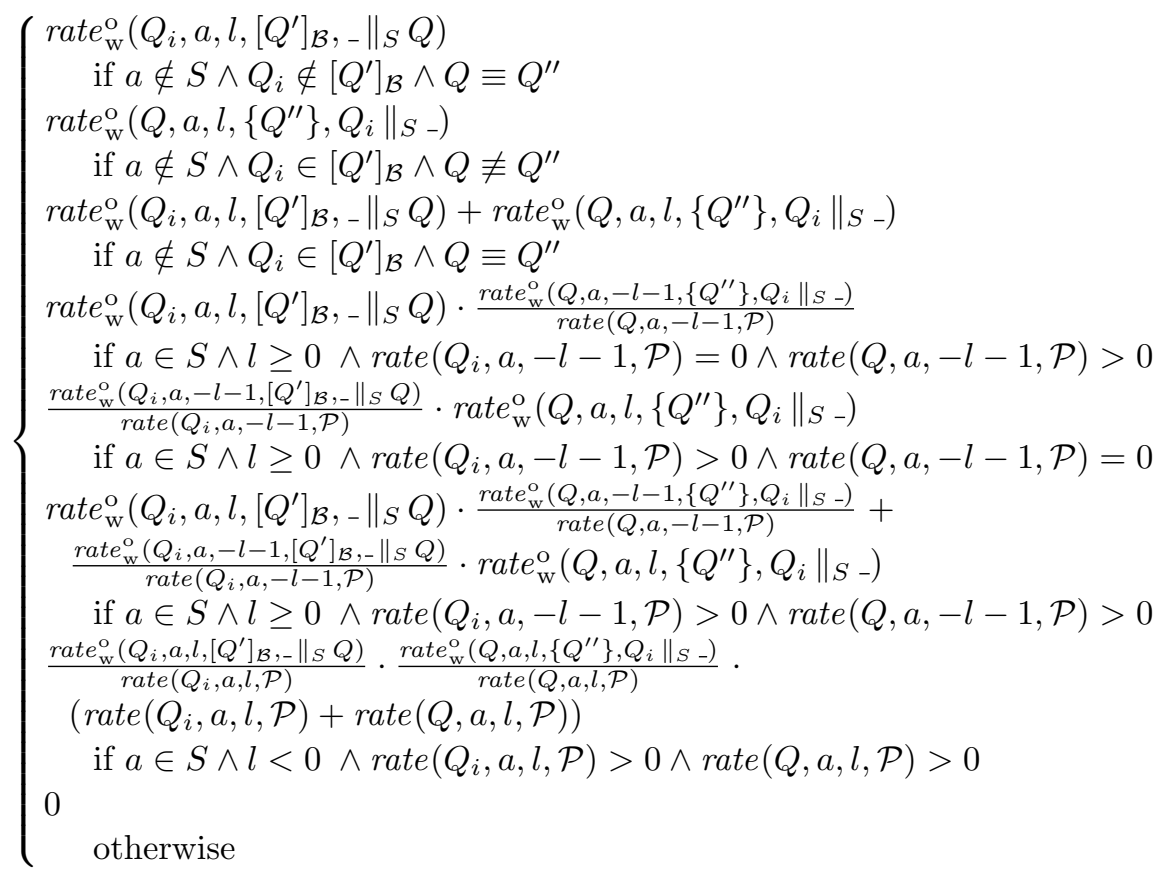

where:

$$
\begin{aligned}
\text { rate }_{\mathrm{w}}^{\mathrm{o}}\left(R, a, l, C,{ }_{-} \|_{S} R^{\prime}\right)= & \operatorname{rate}_{\mathrm{w}}(R, a, l, C)+ \\
& \sum_{\left[R^{\prime \prime}\right]_{\mathcal{B}} \text { obs. s.t. }\left[R^{\prime \prime} \|_{S} R^{\prime}\right]_{\mathcal{B}^{\prime}} \text { unobs. }} \text { rate }_{\mathrm{w}}\left(R, a, l,\left[R^{\prime \prime}\right]_{\mathcal{B}}\right) \cdot \operatorname{prob}_{\mathrm{w}}\left(R^{\prime \prime} \|_{S} R^{\prime}, C\right)
\end{aligned}
$$

is the overall weak exit rate, which takes into account observable states of the individual process terms that become unobservable when applying - $\|_{S}$ - because of synchronization constraints that disable non-pre-emptable visible actions.

From $\left(Q_{1}\left\|_{S} Q, Q_{2}\right\|_{S} Q\right) \in \mathcal{B}^{\prime}$, it follows that $\left(Q_{1}, Q_{2}\right) \in \mathcal{B}$. Since $\mathcal{B}$ is a weak extended Markovian bisimulation, we have that:

$$
\text { rate }_{\mathrm{w}}\left(Q_{1}, a, l,\left[Q^{\prime}\right]_{\mathcal{B}}\right)=\text { rate }_{\mathrm{w}}\left(Q_{2}, a, l,\left[Q^{\prime}\right]_{\mathcal{B}}\right)
$$

and:

$$
\text { rate }_{\mathrm{w}}\left(Q_{1}, a, l,\left[R^{\prime \prime}\right]_{\mathcal{B}}\right)=\text { rate }_{\mathrm{w}}\left(Q_{2}, a, l,\left[R^{\prime \prime}\right]_{\mathcal{B}}\right)
$$

for any observable class $\left[R^{\prime \prime}\right]_{\mathcal{B}}$ such that $\left[R^{\prime} \|_{S} R^{\prime \prime}\right]_{\mathcal{B}^{\prime}}$ is unobservable. Furthermore, by virtue of Lemma A.7, for all $l \in \mathbb{Z}_{<0}$ such that no-pre $\left(l, Q_{1}\right)$ and no-pre $\left(l, Q_{2}\right)$ it holds: 


$$
\operatorname{rate}\left(Q_{1}, a, l, \mathcal{P}\right)=\operatorname{rate}\left(Q_{2}, a, l, \mathcal{P}\right)
$$

The first part of the result then follows.

The case $C=\mathcal{P}_{\text {fu }}$ can be treated similarly to the previous one after observing that the weak exit rate towards $\mathcal{P}_{\text {fu }}$ is the sum of the weak exit rate towards the set $\mathcal{P}_{\text {up }}$ of the states that are part of unescapable paths and of the weak exit rate towards the set $\mathcal{P}_{\text {ts }}$ of the terminal states (like $\underline{0}$ ). Since neither $Q_{1}$ nor $Q_{2}$ can have both unescapable paths and terminal states, for each of them the above mentioned sum will have at most one non-zero summand.

Proof of Thm. 3.3. Let us start with soundness. Since $\approx_{\mathrm{EMB}}$ is an equivalence relation by definition and a congruence over $\mathcal{P}_{\text {wp }, \mathrm{nr}}$ with respect to all the operators by virtue of Cor. 3.1, in the proof of $\mathcal{A} \vdash P_{1}=P_{2}$ it is correct to use reflexivity, symmetry, transitivity, and substitutivity with respect to all the operators.

As far as the set $\mathcal{A}$ of specific axioms is concerned, from Lemma A.5 it follows that $\approx_{\mathrm{EMB}}$ inherits all the axioms of $\sim_{\mathrm{EMB}}$, hence $\mathcal{A}_{1}-\mathcal{A}_{9}$ and $\mathcal{A}_{13}-\mathcal{A}_{20}$ are certainly correct for $\approx_{\mathrm{EMB}}$. The correctness of $\mathcal{A}_{10}-\mathcal{A}_{12}$ stems from the fact that, denoted by $l^{\prime}$ the priority level of the $a$-action and by $Q_{1}$ (resp. $Q_{2}$ ) the process term occurring in the left-hand (resp. right-hand) side of the axiom, after performing the $a$-action both $Q_{1}$ and $Q_{2}$ can reach only $P_{i}$ for $i \in I$ with:

$$
\operatorname{rate}_{\mathrm{w}}\left(Q_{1}, a, l^{\prime},\left\{P_{i}\right\}\right)=\operatorname{rate}\left(Q_{1}, a, l^{\prime}, \mathcal{P}\right) \cdot w_{i} / \sum_{k \in I} w_{k}=\operatorname{rate}_{\mathrm{w}}\left(Q_{2}, a, l^{\prime},\left\{P_{i}\right\}\right)
$$

As far as completeness is concerned, given $P \in \mathcal{P}_{\mathrm{wp}, \mathrm{nr}}$ we say that $P$ is in minimal sum normal form (msnf) iff $P \equiv \underline{0}$ or $P \equiv \sum_{i \in I}<a_{i}, \tilde{\lambda}_{i}>$. $P_{i}$ with $I$ finite and non-empty, $P$ initially minimal with respect to $\mathcal{A}_{4}-\mathcal{A}_{12}$, and $P_{i}$ in msnf for all $i \in I$. In this inductive definition, by initial minimality of $P$ with respect to $\mathcal{A}_{4}-\mathcal{A}_{12}$ we mean that no subset of summands of $P$ matches the left-hand side term of $\mathcal{A}_{4}-\mathcal{A}_{12}$. From the definition it follows that initial minimality holds with respect to $\mathcal{A}_{3}$ as well. We denote by $\mathcal{P}_{\mathrm{wp}, \mathrm{nr}, \mathrm{msfn}}$ the set of the non-recursive well-prioritized process terms that are in msnf. Let us preliminarily prove that for all $P \in \mathcal{P}_{\mathrm{wp}, \mathrm{nr}}$ there exists $Q \in$ $\mathcal{P}_{\text {wp,nr,msfn }}$ such that:

$$
\mathcal{A} \vdash P=Q
$$

by proceeding by induction on the syntactical structure of the non-recursive process term $P$ : 
- If $P \equiv \underline{0}$, the result follows by taking $Q \equiv \underline{0}$ (which is in msnf) and using reflexivity.

- If $P \equiv<a, \tilde{\lambda}>\cdot P^{\prime}$, then by the induction hypothesis there exists $Q^{\prime}$ in msnf such that $\mathcal{A} \vdash P^{\prime}=Q^{\prime}$. From substitutivity with respect to action prefix we obtain that $\mathcal{A} \vdash<a, \tilde{\lambda}>. P^{\prime}=<a, \tilde{\lambda}>$. $Q^{\prime}$, from which the result follows as $<a, \tilde{\lambda}>$. $Q^{\prime}$ is in msnf.

- If $P \equiv P_{1}+P_{2}$, then by the induction hypothesis there exist $Q_{1}$ and $Q_{2}$ in msnf such that $\mathcal{A} \vdash P_{1}=Q_{1}$ and $\mathcal{A} \vdash P_{2}=Q_{2}$. From substitutivity with respect to alternative composition we obtain that $\mathcal{A} \vdash P_{1}+P_{2}=$ $Q_{1}+Q_{2}$. If $Q_{1}+Q_{2}$ is in msnf, we are done. If instead $Q_{1}+Q_{2}$ is not in msnf - because it is not initially minimal with respect to $\mathcal{A}_{3}-\mathcal{A}_{9}$ - the result follows after as many applications of $\mathcal{A}_{3}-\mathcal{A}_{9}$ to $Q_{1}+Q_{2}$ as possible (possibly preceded by applications of $\mathcal{A}_{1}-\mathcal{A}_{2}$ ) by virtue of substitutivity with respect to alternative composition as well as transitivity.

- If $P \equiv P^{\prime} / L$, then by the induction hypothesis there exists $Q^{\prime}$ in msnf such that $\mathcal{A} \vdash P^{\prime}=Q^{\prime}$. From substitutivity with respect to hiding we obtain that $\mathcal{A} \vdash P^{\prime} / L=Q^{\prime} / L$. The result follows after as many applications of $\mathcal{A}_{13}-\mathcal{A}_{16}$ to $Q^{\prime} / L$ as possible (possibly interleaved with applications of $\mathcal{A}_{4}-\mathcal{A}_{12}$ preceded by applications of $\mathcal{A}_{1}-\mathcal{A}_{2}$ ) by virtue of substitutivity with respect to action prefix and alternative composition as well as transitivity.

- If $P \equiv P^{\prime}[\varphi]$, then by the induction hypothesis there exists $Q^{\prime}$ in msnf such that $\mathcal{A} \vdash P^{\prime}=Q^{\prime}$. From substitutivity with respect to relabeling we obtain that $\mathcal{A} \vdash P^{\prime}[\varphi]=Q^{\prime}[\varphi]$. The result follows after as many applications of $\mathcal{A}_{17}-\mathcal{A}_{19}$ to $Q^{\prime}[\varphi]$ as possible (possibly interleaved with applications of $\mathcal{A}_{4}-\mathcal{A}_{6}$ preceded by applications of $\mathcal{A}_{1}-\mathcal{A}_{2}$ ) by virtue of substitutivity with respect to action prefix and alternative composition as well as transitivity.

- If $P \equiv P_{1} \|_{S} P_{2}$, then by the induction hypothesis there exist $Q_{1}$ and $Q_{2}$ in msnf such that $\mathcal{A} \vdash P_{1}=Q_{1}$ and $\mathcal{A} \vdash P_{2}=Q_{2}$. From substitutivity with respect to parallel composition we obtain that $\mathcal{A} \vdash P_{1}\left\|_{S} P_{2}=Q_{1}\right\|_{S} Q_{2}$. The result follows after as many applications of $\mathcal{A}_{20}$ to $Q_{1} \|_{S} Q_{2}$ as possible (possibly interleaved with applications of $\mathcal{A}_{4}-\mathcal{A}_{12}$ preceded by applications of $\mathcal{A}_{1}-\mathcal{A}_{2}$ ) by virtue of substitutivity with respect to action prefix and alternative composition as well as transitivity.

Now there are two cases. If $P_{1}$ and $P_{2}$ are both in msnf, to prove completeness we proceed by induction on the syntactical structure of $P_{1}$ : 
- If $P_{1} \equiv \underline{0}$, from $P_{1} \approx_{\mathrm{EMB}} P_{2}$ and $P_{2}$ in msnf it follows that $P_{2} \equiv \underline{0}$, hence the result by reflexivity.

- If $P_{1} \equiv \sum_{i \in I_{1}}<a_{i}, \tilde{\lambda}_{i}>\cdot P_{1, i}$ with $I_{1}$ finite and non-empty, from $P_{1} \approx_{\mathrm{EMB}} P_{2}$ and $P_{2}$ in msnf it follows that $P_{2} \equiv \sum_{j \in I_{2}}<b_{j}, \tilde{\mu}_{j}>\cdot P_{2, j}$ with $I_{2}$ finite and non-empty. We note that:

- Neither $P_{1}$ nor $P_{2}$ admits unobservable paths - if we exclude possible initial $\tau$-transitions - due to the initial minimality of $P_{1}, P_{2}$, and their derivatives with respect to $\mathcal{A}_{10}-\mathcal{A}_{12}$.

- None of the actions occurring in $P_{1}$ and $P_{2}$ can be pre-empted due to the initial minimality of $P_{1}, P_{2}$, and their derivatives with respect to $\mathcal{A}_{7}-\mathcal{A}_{9}$.

By virtue of Lemma A.7, from $P_{1} \approx_{\mathrm{EMB}} P_{2}$ we derive that:

$$
\left\{a_{i} \mid i \in I_{1}\right\}=\left\{b_{j} \mid j \in I_{2}\right\} \equiv\left\{c_{1}, c_{2}, \ldots, c_{n}\right\}
$$

with:

$$
\operatorname{rate}\left(P_{1}, c_{k}, l, \mathcal{P}\right)=\operatorname{rate}\left(P_{2}, c_{k}, l, \mathcal{P}\right)
$$

for each $k=1, \ldots, n$ and all $l \in \mathbb{Z}$ such that no-pre $\left(l, P_{1}\right)$ and no-pre $\left(l, P_{2}\right)$. We can then concentrate on a generic $c_{k}$ at a priority level $l$ such that $\operatorname{rate}\left(P_{1}, c_{k}, l, \mathcal{P}\right)=\operatorname{rate}\left(P_{2}, c_{k}, l, \mathcal{P}\right)>0$ and on the two related sets of summands:

$S_{k, 1}=\left\{<a_{i}, \tilde{\lambda}_{i}>\cdot P_{1, i} \mid i \in I_{1} \wedge a_{i}=c_{k} \wedge \tilde{\lambda}_{i}\right.$ has priority $\left.l\right\}$

$S_{k, 2}=\left\{<b_{j}, \tilde{\mu}_{j}>\cdot P_{2, j} \mid j \in I_{2} \wedge b_{j}=c_{k} \wedge \tilde{\mu}_{j}\right.$ has priority $\left.l\right\}$ which satisfy the following two properties:

(1) $\sum_{P \in S_{k, 1}} \operatorname{rate}\left(P, c_{k}, l, \mathcal{P}\right)=\sum_{P \in S_{k, 2}} \operatorname{rate}\left(P, c_{k}, l, \mathcal{P}\right)$.

(2) The derivative terms $P_{1, i}$ (resp. $P_{2, j}$ ) occurring in $S_{k, 1}$ (resp. $S_{k, 2}$ ) are all inequivalent with respect to $\approx_{\mathrm{EMB}}$ due to the initial minimality of $P_{1}$ (resp. $P_{2}$ ) with respect to $\mathcal{A}_{4}-\mathcal{A}_{6}$. In fact, due to such an initial minimality, taken two derivative terms in the same summand set, it must be the case that their sets of initial action names are different or the total exit rate with respect to one of these initial action names is different in the two derivative terms, thus violating the necessary condition for $\approx_{\mathrm{EMB}}$ stated by Lemma A.7.

We now prove by proceeding by induction on $\left|S_{k, 1}\right|$ that for all summands $<a_{i}, \tilde{\lambda}_{i}>\cdot P_{1, i} \in S_{k, 1}$ there exists exactly one summand $<b_{j}, \tilde{\mu}_{j}>. P_{2, j} \in S_{k, 2}$ such that $\tilde{\lambda}_{i}=\tilde{\mu}_{j} \wedge P_{1, i} \approx_{\mathrm{EMB}} P_{2, j}$ :

- If $\left|S_{k, 1}\right|=1$, then $S_{k, 1}$ contains a single summand, say $<a_{i}, \tilde{\lambda}_{i}>\cdot P_{1, i}$. Then $S_{k, 2}$ must contain a single summand as well, say $<b_{j}, \tilde{\mu}_{j}>\cdot P_{2, j}$, with $\tilde{\lambda}_{i}=\tilde{\mu}_{j} \wedge P_{1, i} \approx_{\mathrm{EMB}} P_{2, j}$ as $P_{1} \approx_{\mathrm{EMB}} P_{2}$. The reason why $S_{k, 2}$ cannot contain several summands is that, by 
virtue of $P_{1} \approx_{\mathrm{EMB}} P_{2}$, the derivative of each of them should be weakly extended Markovian bisimilar to $P_{1, i}$, which would contra$\operatorname{dict}(2)$ for $P_{2}$.

- Suppose that $\left|S_{k, 1}\right|=m>1$. Let $Q$ be one of the derivative terms occurring in $S_{k, 1}$. By virtue of (2) there is only one summand in $S_{k, 1}$ that reaches $[Q]_{\approx_{\mathrm{EMB}}}$ when executing actions with name $c_{k}$ and priority level $l$. Since $P_{1} \approx_{\mathrm{EMB}} P_{2}$, there must exist a summand in $S_{k, 2}$ that reaches $[Q]_{\approx_{\mathrm{EMB}}}$ when executing actions with name $c_{k}$ and priority level $l$. Since no other summand of $S_{k, 2}$ can reach $[Q]_{\approx_{\mathrm{EMB}}}$ when executing actions with name $c_{k}$ and priority level $l$ by virtue of (2), the rates of the two initial actions of the considered summands must coincide.

Let us now denote by $S_{k, 1}^{\prime}$ and $S_{k, 2}^{\prime}$ the two sets obtained from $S_{k, 1}$ and $S_{k, 2}$, respectively, after removing the two considered summands. Since $S_{k, 1}^{\prime}$ and $S_{k, 2}^{\prime}$ satisfy (1) and (2) with $\left|S_{k, 1}^{\prime}\right|=$ $m-1$, by the induction hypothesis it follows that for all summands $<a_{i}, \tilde{\lambda}_{i}>\cdot P_{1, i} \in S_{k, 1}^{\prime}$ there exists exactly one summand $<b_{j}, \tilde{\mu}_{j}>\cdot P_{2, j} \in S_{k, 2}^{\prime}$ such that $\tilde{\lambda}_{i}=\tilde{\mu}_{j} \wedge P_{1, i} \approx_{\mathrm{EMB}} P_{2, j}$. Thus the result follows for the whole $S_{k, 1}$ and $S_{k, 2}$.

By proceeding in a similar way we can prove that for all summands $<b_{j}, \tilde{\mu}_{j}>. P_{2, j} \in S_{k, 2}$ there exists exactly one summand $<a_{i}, \tilde{\lambda}_{i}>. P_{1, i} \in$ $S_{k, 1}$ such that $\tilde{\lambda}_{i}=\tilde{\mu}_{j} \wedge P_{1, i} \approx_{\mathrm{EMB}} P_{2, j}$.

As a consequence, a bijective correspondence can be established between $S_{k, 1}$ and $S_{k, 2}$. Let us take a pair of corresponding summands $<a_{i}, \tilde{\lambda}_{i}>\cdot P_{1, i}$ and $<b_{j}, \tilde{\mu}_{j}>\cdot P_{2, j}$, so that $a_{i}=b_{j} \wedge \tilde{\lambda}_{i}=\tilde{\mu}_{j} \wedge P_{1, i} \approx_{\mathrm{EMB}}$ $P_{2, j}$. Since $P_{1, i}$ and $P_{2, j}$ are in msnf, by the induction hypothesis it follows that $\mathcal{A} \vdash P_{1, i}=P_{2, j}$, hence $\mathcal{A} \vdash<a_{i}, \tilde{\lambda}_{i}>. P_{1, i}=$ $<b_{j}, \tilde{\mu}_{j}>\cdot P_{2, j}$ by substitutivity with respect to action prefix, hence $\mathcal{A} \vdash \sum_{i \in I}<a_{i}, \tilde{\lambda}_{i}>. P_{1, i}=\sum_{j \in J}<b_{j}, \mu_{j}>. P_{2, j}$ by substitutivity with respect to alternative composition and the bijectivity of the correspondence.

If instead it is not the case that $P_{1}$ and $P_{2}$ are both in msnf, we exploit the fact that there exist $Q_{1}$ and $Q_{2}$ in msnf such that $\mathcal{A} \vdash P_{1}=Q_{1}$ and $\mathcal{A} \vdash P_{2}=Q_{2}$. From soundness it follows that $P_{1} \approx_{\mathrm{EMB}} Q_{1}$ and $P_{2} \approx_{\mathrm{EMB}}$ $Q_{2}$, hence $Q_{1} \approx_{\mathrm{EMB}} Q_{2}$ since $P_{1} \approx_{\mathrm{EMB}} P_{2}$ by the initial hypothesis and $\approx_{\mathrm{EMB}}$ is a transitive relation. Since $Q_{1}$ and $Q_{2}$ are both in msnf, from what demonstrated in the previous part of the proof of completeness we derive that $\mathcal{A} \vdash Q_{1}=Q_{2}$, hence $\mathcal{A} \vdash P_{1}=P_{2}$ by transitivity. 